Pacific Northwest

National Laboratory

Operated by Battelle for the

U.S. Department of Energy

\title{
FY07 Final Report for Calibration Systems
}

TL Myers

BT Broocks

BD Cannon

$\mathrm{N}$ Ho

December 2007

Prepared for the U.S. Department of Energy under Contract DE-AC05-76RL01830 


\title{
DISCLAIMER
}

This report was prepared as an account of work sponsored by an agency of the United States Government. Neither the United States Government nor any agency thereof, nor Battelle Memorial Institute, nor any of their employees, makes any warranty, express or implied, or assumes any legal liability or responsibility for the accuracy, completeness, or usefulness of any information, apparatus, product, or process diselosed, or represents that its use would not infringe privately owned rights. Reference herein to any specific commercial product, process, or service by trade name, trademark, manufacturer, or otherwise does not necessarily constitute or imply its endorsement, recommendation, or favoring by the United States Government or any agency thereof, or Battelle Memorial Institute. The views and opinions of authors expressed herein do not necessarily state or reflect those of the United States Government or any agency thereof.

\author{
PACIFIC NORTHWEST NATIONAL LABORATORY \\ operated by \\ BATTELLE \\ for the \\ UNITED STATES DEPARTMENT OF ENERGY \\ under Contract DE-AC05-76RL01830 \\ Printed in the United States of America \\ Available to DOE and DOE contractors from the \\ Office of Scientific and Technical Information, \\ P.O. Box 62, Oak Ridge, TN 37831-0062; \\ ph: (865) 576-8401 \\ fax: (865) 576-5728 \\ email: reports@adonis.osti.gov \\ Available to the public from the National Technical Information Service, \\ U.S. Department of Commerce, 5285 Port Royal Rd., Springfield, VA 22161 \\ ph: (800) 553-6847 \\ fax: (703) 605-6900 \\ email: orders@ntis.fedworld.gov \\ online ordering: http://www.ntis.gov/ordering.htm
}

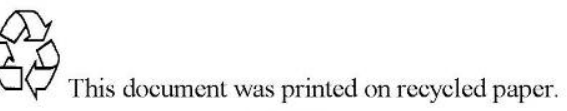

$(9 / 2003)$ 


\title{
FY07 Final Report for Calibration Systems
}

\author{
TL Myers \\ BT Broocks \\ BD Cannon \\ N Hô
}

December 2007

Prepared for

the U.S. Department of Energy

under Contract DE-AC05-76RL01830

Pacific Northwest National Laboratory

Richland, Washington 99352 


\section{Summary}

Remote infrared (IR) sensing provides a valuable method for detection and identification of materials associated with nuclear proliferation. Current challenges for remote sensors include minimizing the size, mass, and power requirements for cheaper, smaller, and more deployable instruments without affecting the measurement performance. One area that is often overlooked is sensor calibration design that is optimized to minimize the cost, size, weight, and power of the payload. Yet, an on-board calibration system is essential to account for changes in the detector response once the instrument has been removed from the laboratory. The Calibration Systems project at Pacific Northwest National Laboratory (PNNL) is aimed towards developing and demonstrating compact quantum cascade (QC) laser-based calibration systems for infrared sensor systems in order to provide both a spectral and radiometric calibration while minimizing the impact on the instrument payload.

In FY05, PNNL demonstrated a multi-level radiance scheme that provides six radiance levels for an enhanced linearity check compared to the currently accepted two-point scheme. PNNL began testing the repeatability of this scheme using a cryogenically cooled, single-mode quantum cascade laser (QCL). A cyclic variation in the power was observed that was attributed to the thermal cycling of the laser's dewar. In FY06, PNNL continued testing this scheme and installed an auxiliary liquid nitrogen reservoir to limit the thermal cycling effects. Although better repeatability was achieved over a longer time period, power fluctuations were still observed due to the thermal cycling.

Due to the limitations with the cryogenic system, PNNL began testing Fabry-Perot QCLs that operate continuous-wave (cw) or quasi-cw at room temperature (RT) in FY06. PNNL demonstrated a multi-level scheme that provides five radiance levels in 105 seconds with excellent repeatability. We have continued testing this repeatability in FY07. A burn-in effect appears in which the power increases over a certain time period. Repeatability better than $1 \%$, however, is demonstrated for most of the radiance levels after this initial burn-in.

In FY06, PNNL also began investigating a fiber-coupled RT QCL for a compact IR calibration source. PNNL demonstrated a uniform beam profile by measuring a time-averaged response and modulating the fiber optic with a motor to minimize the effects of speckle. In FY07, PNNL examined the power stability of fiber-coupled QCLs. Feedback appears to degrade the stability so that anti-reflective coatings for fibers may be essential.

In FY07, PNNL continued to investigate the stability of room temperature QCLs as well as the measurement technique to provide a quantitative estimate for the measurement uncertainty. We designed and built a custom environmental enclosure to reduce the measurement uncertainty. After an initial burnin, we have achieved uncertainties better than $0.1 \%$ for data collected over almost 100 hours of operation. We also built a bench-top system to demonstrate how the QC laser can be used to calibrate a microbolometer array and illustrated the importance of a multi-point calibration. 


\section{Acronyms}

$\begin{array}{ll}\text { Cw } & \text { continuous-wave } \\ \text { DAC } & \text { digital-to-analog converter } \\ \text { HVAC } & \text { heating, ventilation, and air conditioning } \\ \text { IR } & \text { infrared } \\ \text { L-I } & \text { light versus current } \\ \text { PNNL } & \text { Pacific Northwest National Laboratory } \\ \text { QC } & \text { quantum cascade } \\ \text { QCL } & \text { quantum cascade laser } \\ \text { RT } & \text { room temperature } \\ \text { TEC } & \text { thermo-electric cooler }\end{array}$




\section{Contents}

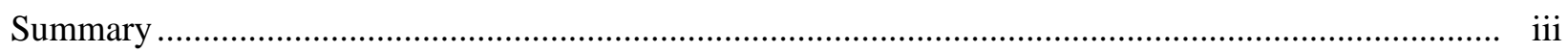

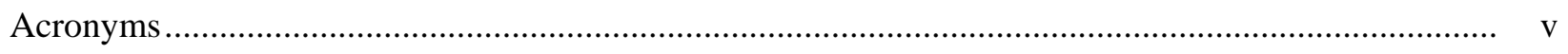

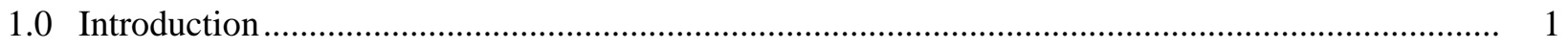

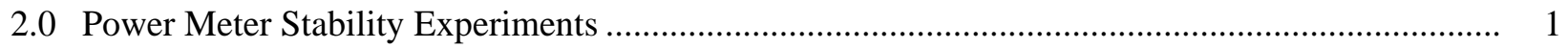

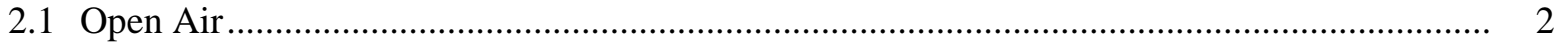

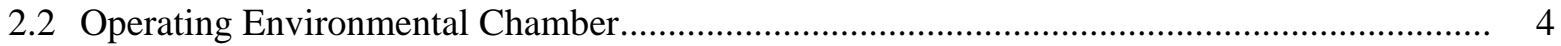

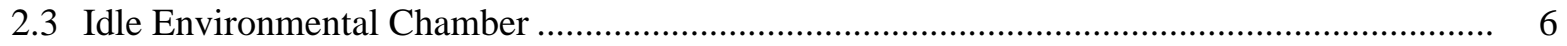

2.4 Inexpensive Custom Environmental Enclosure ............................................................... 9

2.5 3-mW Scale and TEC Running in Custom Environmental Enclosure .................................... 11

3.0 Stability of QCL in Custom Environmental Enclosure.............................................................. 13

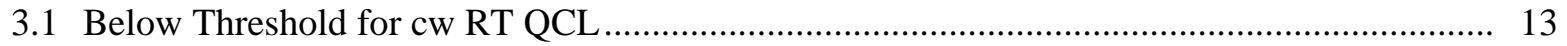

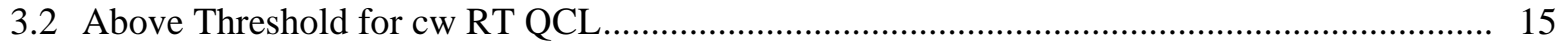

3.3 Above Threshold for QCL with Modulated Currents …....................................................... 17

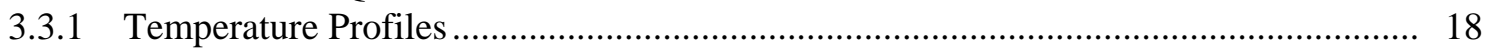

3.3.2 Long-term Measurements ......................................................................... 18

4.0 Stability of QCL in Open Air with Modulated Currents........................................................... 20

5.0 Stability of Mid-Infrared Fiber Transmission ......................................................................... 23

6.0 Calibration System for a Microbolometer Array …................................................................ 25

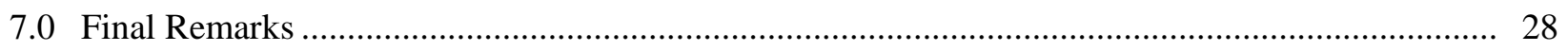

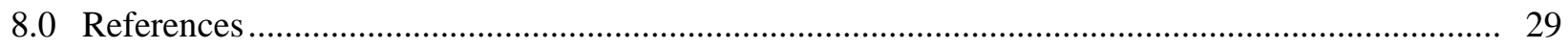




\section{Figures}

1 Initial and Final Zero Readings from the Power Meter in the Laboratory in Open Air

2 Power Deviations from the Mean Using Initial Zero Subtraction and a Linear Interpolation Zero Subtraction for the Measurements in Open Air.

3 Initial and Final Zero Readings from the Power Meter in the Environmental Chamber at $30.0^{\circ} \mathrm{C}$

4 Power Deviations from the Mean Using Initial Zero Subtraction and a Linear Interpolation Zero Subtraction for the Measurements in the Environmental Chamber at $30.0^{\circ} \mathrm{C}$

5 Initial and Final Zero Power Readings from the Power Meter in the Idle Environmental Chamber

6 Power Deviations from the Mean Using Initial Zero Subtraction and a Linear Interpolation Zero Subtraction for the Measurements in an Idle Environmental Chamber

7 Power Deviations from the Mean Using Initial Zero Subtraction for the Measurements in the Idle Environmental Chamber

8 Simple Environmental Enclosure for Stability Testing.......

9 Initial and Final Zero Power Readings from the Power Meter in the Custom

Environmental Chamber

10 Power Deviations from the Mean Using an Interpolated Zero Subtraction for the Measurements in the Custom Environmental Chamber

11 Initial and Final Zero Power Readings from the Power Meter in the Custom Environmental Chamber with a Resolution of $0.001 \mathrm{~mW}$

12 Power Deviations from the Mean Using Initial Zero Subtraction for the Measurements in the Custom Environmental Chamber.

13 Power Meter Readings for All Six Steps Below Threshold in the Custom Environmental Chamber....

14 Power Deviations from the Mean Using Initial Zero Subtraction for the Five Steps at Different Currents, which are All below Threshold

15 Power Deviations from the Mean Using Initial Zero Subtraction at Five Different Current Levels for a QC Laser that is Operated cw in the Custom Environmental Chamber.

16 Expanded View of Variations in Power Meter Readings from Their Means for the Five Above Threshold Steps Shown in Figure 15 
17 Power Deviations from the Mean Using Initial Zero Subtraction at Five Different Current Levels for a QC Laser Using a 40 kHz, 50\% Duty Cycle Current Modulation in the Custom Environmental Chamber.

18 Temperature Profiles During a Single Cycle with DC Current Steps and a $40 \mathrm{kHz}$ Modulated 50\% Duty Cycle Current Steps

19 Power Deviations from the Mean Using Initial Zero Subtraction at Five Different Current Levels for a QC Laser Using a $40 \mathrm{kHz}, 50 \%$ Duty Cycle Current Modulation in the Custom Environmental Chamber

20 Initial Zero Power Readings from the Power Meter in the Open Air

21 Power Deviations from the Mean Using Initial Zero Subtraction at Five Different Current Levels for a QC Laser Using a $100 \mathrm{kHz}, 10 \%$ Duty Cycle Current Modulation in Open Air Over Three Weeks

22 Power Deviations from the Mean Using Initial Zero Subtraction at Five Different Current Levels for a QC Laser Using a 100 kHz, 10\% Duty Cycle Current Modulation in Open Air Over Ten Months

23 Photographs of the Multimode Chalcogenide Glass Optical Fiber Coupled to the QCL and the Motor Used to Move the Middle of the Fiber Up and Down to Mix the Propagating Mode and Create a Uniform Output Beam

24 Typical Image from the Calibration System

25 Distribution of Calibration Coefficients for Selected Pixels.

26 Time Evolution of the Calibration Coefficients

\section{Tables}

1 Power and Relative Confidence Limits of Power Meter in Open Air for 1000 Cycles using Both a) an Initial Zero Subtraction and b) a Linear Interpolation.....

2 Power and Relative Confidence Limits of Power Meter Head in Operating Environmental Chamber at $30.0^{\circ} \mathrm{C}$ for 500 Cycles Using Both a) an Initial Zero Subtraction and b) a Linear Interpolation

3 Power and Relative Confidence Limits of Power Meter Head in Idle Environmental Chamber for 472 Cycles Using Both a) an Initial Zero Subtraction and b) a Linear Interpolation.

4 Power and Relative Confidence Limits of Power Meter Head in Idle Environmental Chamber for 2172 Cycles Using Both a) an Initial Zero Subtraction and b) a Linear Interpolation. 
5 Power and Relative Confidence Limits of Power Meter Head in Custom Environmental Chamber for 2525 Cycles Using Both a) an Initial Zero Subtraction and b) a Linear Interpolation

6 Power and Relative Confidence Limits of Power Meter Head in Custom Environmental Enclosure for 240 Cycles Using an Initial Zero Subtraction and the 3-mW Scale on the Power Meter.

7 Zero Subtracted Power Meter Readings and Standard Deviations for Five Different Current Levels Applied to a QCL in a Custom Environmental Enclosure Along with the Power Meter Head over 429 Cycles

8 Zero Subtracted Power Meter Readings and Standard Deviations for Five Different DC Current Waveforms Applied to a QCL in the Custom Enclosure for 160 Cycles

9 Zero Subtracted Power Meter Readings and Standard Deviations for Five Different Modulated Current Waveforms Applied to a QCL in a Custom Environmental Enclosure

10 Zero Subtracted Power Meter Readings and Standard Deviations for the Data Shown in Figure 19

11 Zero Subtracted Power Meter Readings and Standard Deviations for the Data Shown in Figure 19 Excluding the First 900 Cycles....

12 Zero Subtracted Power Meter Readings and Standard Deviations for the Data Shown in Figure 21

13 Zero Subtracted Power Meter Readings and Standard Deviations for the Data Shown in Figure 22

14 Time Constants from the Exponential Fits to the Data Shown in Figure 22 as Well as the Average Power from the First 3000 Cycles.

15 Zero Subtracted Power Meter Readings and Standard Deviations for the Data Shown in Figure 22 After Three Time Constants

16 Laser Amplitude Noise for Different Conditions .......................................................................25

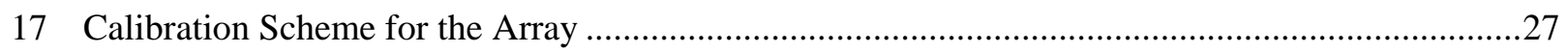

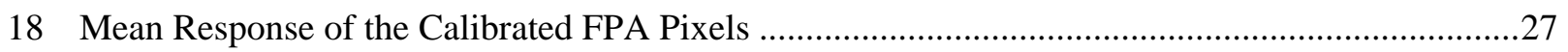




\subsection{Introduction}

Accurate radiometric measurements require a comprehensive characterization of the measuring instrument including the fore-optics and the detectors. Prior to deployment, an instrument can be accurately and completely characterized, but as changes occur after deployment, such as spectral response drifts and thermal fluctuations, on-board calibration checks are necessary. Most on-board calibrators in the infrared provide a limited two-point linearity check and do not monitor the spectral response, which is generally assumed not to change from pre-deployment laboratory calibration.

Quantum cascade lasers are semiconductor devices that emit in the infrared and offer several advantages as calibration sources such as a fast time response and radiometric and spectral stability. Photon generation in a quantum cascade (QC) laser results from an electronic transition between states that are created by quantum confinement effects in the quantum wells. Thus, QC lasers can be tailored to emit light at a specific wavelength throughout the infrared region $(3.5-17 \mu \mathrm{m})$ because the thickness of the quantum wells determines the wavelength. Furthermore, a single electron emits multiple photons as it undergoes multiple transitions among a series of quantum wells so that higher optical powers result. Another feature of QC lasers is that they are unipolar devices, for they rely on only one type of carrier (i.e., electrons) making them more robust than typical diode lasers, which are sensitive to electrostatic discharge. In addition, a grating can be incorporated into the structure so that the laser emits from a single longitudinal mode and provides a narrow spectral linewidth. In the past few years, attempts to improve the QC designs (lower optical losses and improve population inversion at room temperature [RT]) and to improve the heat conduction (epi-down mounting, buried heterostructure designs, InP cladding, and thick gold) have culminated in QC lasers that operate continuous wave (cw) or quasi-cw at RT with high output power. Thus, QC lasers have superb power and spectral stability and also offer the ability to operate either pulsed or $\mathrm{cw}$ at high operating temperatures.

For wavelength calibration, a set of lasing wavelengths can be chosen that are spread evenly across the entire spectral range of the instrument to be calibrated and cover the edges of the spectral range to provide the needed sub-pixel accuracy. Since the output power can be adjusted rapidly to span the dynamic range of the detector, QC lasers can provide a nonlinearity correction on a fast timescale by moving beyond a two-point calibration scheme. Thus, QC lasers are a viable calibration source and can provide a useful complement to blackbodies to improve the overall characterization and calibration of infrared instruments.

\subsection{Power Meter Stability Experiments}

The long-term measurements of the quantum cascade laser (QCL) power stability are performed using power meters manufactured by Coherent. The power meter includes a thermopile sensor and a readout module with an RS-232 serial connection for data transfer to a computer. The observed stability is the combined stability of the QCL under test, the power meter and its readout, and the stability of the parts of the data acquisition system including the digital-to-analog converter (DAC) used to set the current for the QCL and the current supply for the QCL. In particular, the power meter and its readout may be the limiting factor in the observed stability. To test this possibility, we conducted experiments to 
determine the power meter stability and to establish optimum conditions for stability independent of QCL performance.

For these experiments, the thermopile sensor was excited by electrically heating a very small and thermally isolated resistor that was held a few millimeters from the sensitive surface of the thermopile. To change the measurement on the thermopile, the voltage from the DAC applied to the resistor is changed. This configuration eliminated the current supply, the QCL, and the system controlling the temperature of the QCL from these tests of the power meter stability. The heating appears to be very reproducible, and the power meter readings are similar to those produced in some of the QCL stability tests. The readout module for the power meter is maintained on a scale that measures a maximum power of $30 \mathrm{~mW}$ and provides a resolution of $0.01 \mathrm{~mW}$.

\subsection{Open Air}

The first measurements were made with the power meter placed on an optical table and open to the room air in an uncontrolled laboratory. Thus, the system is susceptible to air currents and temperature cycling from the heating, ventilation, and air conditioning (HVAC) system. Preliminary experiments demonstrated that it takes about 45 seconds for the power meter reading to stabilize after changing the voltage applied to the resistor; therefore, each cycle included six steps that were 60 seconds long with power meter readings taken every $1 / 2$ second for the last 15 seconds and the results averaged. The cycles were repeated every ten minutes. The first step, R0, and the last step, R5, do not include any voltage through the resistor so that these two steps provide an initial and a final zero power reading, respectively. In our experiments, we use the zero power reading for subtracting out the offset from the power meter to minimize the effects of thermal drifts in the power meter. The intermediate steps, R1 through R4, include voltage applied to the resistor in increasing increments.

Figure 1 shows the initial zero readings, R0, as the black trace and the final zero readings, R5, as the red trace for 1000 cycles, which spans about one week. The mean of the initial zero readings is $22.8 \mu \mathrm{W}$ with a standard deviation of $11.5 \mu \mathrm{W}(22.8 \mu \mathrm{W} \pm 11.5 \mu \mathrm{W})$ whereas the mean of the final zero readings is $88.7 \mu \mathrm{W} \pm 11.0 \mu \mathrm{W}$. Subtraction of the initial and final zero readings yield a mean signal of $66 \mu \mathrm{W} \pm$ $8 \mu \mathrm{W}$. The data reflects that 1 ) the power meter has not fully recovered to a true zero at the end of the cycle, 2) the initial and final zero reading share a source of variation that is removed by subtraction, and 3) the output resolution of the power meter on the $30 \mathrm{~mW}$ scale of .01 $\mathrm{mW}$ is a limiting factor in the initial and final zero readings, which is not removed even with 15 seconds of averaging.

The power meter has not recovered to a true zero at the end of the measurement cycle due to local heating of the absorbing element relative to the bulk of the thermopile during the cycle. Four minutes later, however, at the start of the next cycle, the absorbing element has equilibrated to the bulk temperature. This heating effect is constant for each cycle and just results in an offset between the initial and final zero readings

Because of the long timescale of the experiment (six minutes), variations in the laboratory temperature and air currents will have occurred during the course of a cycle. These changes will not be the same with each cycle but will vary causing subtle differences between the initial and final zero readings. Therefore, improvements may be made by adjusting the method used to subtract the offset from 
the power meter. Figure 2 shows the corrected power for the last step in the cycle using two different subtraction methods. The black trace shows the typical method used when only the initial zero reading is

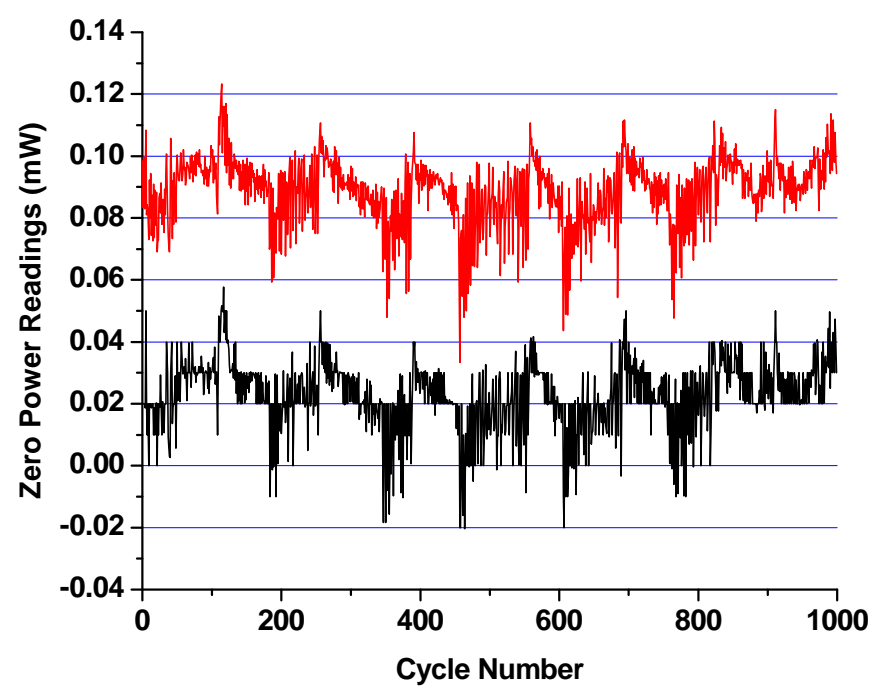

Figure 1. Initial (black) and Final (red) Zero Readings from the Power Meter in the Laboratory in Open Air

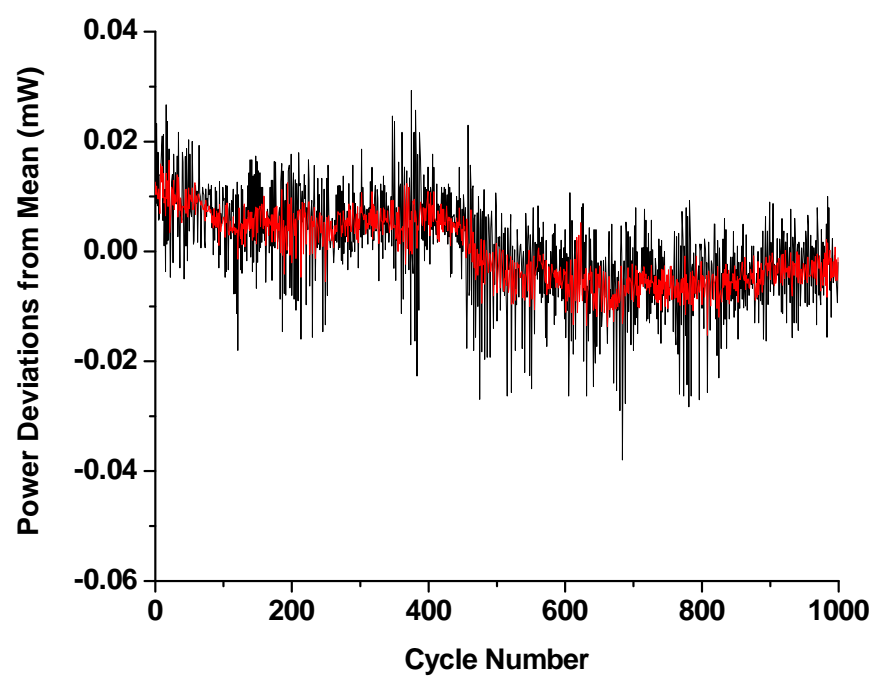

Figure 2. Power Deviations from the Mean Using Initial Zero Subtraction (black) and a Linear Interpolation Zero Subtraction (red) for the Measurements in Open Air

subtracted from the measured value. The red trace shows the value obtained when the zero is subtracted using a linear interpolation between the initial and final zero measurements. The larger variations in the black trace show a simple initial zero subtraction is limited. Lower uncertainty is achieved using a linear interpolation of the zero reading because it can more accurately reflect the change in the zero reading during the course of the measurement. 
Table 1. Power and Relative Confidence Limits of Power Meter in Open Air for 1000 Cycles using Both a) an Initial Zero Subtraction and b) a Linear Interpolation

\begin{tabular}{|l|c|c|c|c||}
\hline \multicolumn{5}{|c||}{ a) Initial Zero Subtraction } \\
\hline Average Power $(\mathrm{mW})$ & 1.103 & 2.201 & 3.265 & 4.278 \\
\hline Standard Deviation $(\mu \mathrm{W})$ & 3.5 & 5.1 & 7.4 & 9.8 \\
\hline $2 \times$ Rel. Std. Dev. & $0.63 \%$ & $0.46 \%$ & $0.45 \%$ & $0.46 \%$ \\
\hline \multicolumn{5}{|c|}{ b) Linear Interpolation Zero Subtraction } \\
\hline Average Power $(\mathrm{mW})$ & 1.089 & 2.174 & 3.226 & 4.226 \\
\hline Standard Deviation $(\mu \mathrm{W})$ & 3.0 & 3.6 & 4.9 & 6.2 \\
\hline $2 \times$ Rel. Std. Dev. & $0.54 \%$ & $0.33 \%$ & $0.30 \%$ & $0.29 \%$ \\
\hline \hline
\end{tabular}

Table 1 lists the average power, standard deviation, and twice the relative standard deviation for all four levels with the zero subtracted using a) the initial zero reading and b) a linear interpolation of the initial and final zero readings. Thus, both Figure 2 and Table 1 demonstrate that a linear interpolation of the initial and final zero readings minimizes the measurement uncertainty. The magnitude of the average power also changes because of the offset between the initial and final zero readings. Although this effect impacts absolute measurements, it does not influence measurements of the reproducibility.

\subsection{Operating Environmental Chamber}

In the next experiment, the thermopile detector with the test resistor was moved into an environmental chamber to provide a more controlled environment by maintaining a constant temperature. The temperature of the chamber was stabilized at $30.0 \pm 0.1^{\circ} \mathrm{C}$. The initial (black trace) and final (red trace) zero readings for this system are shown in Figure 3. The cyclic variations that result from the HVAC are no longer present and the fluctuations appear randomized. The mean of the initial zero readings is $7.4 \mu \mathrm{W} \pm 8.4 \mu \mathrm{W}$ whereas the mean of the final zero readings is $68.7 \mu \mathrm{W} \pm 8.5 \mu \mathrm{W}$. Subtraction of the initial and final zero readings yields a mean signal of $61.2 \mu \mathrm{W} \pm 12.2 \mu \mathrm{W}$ where the larger standard deviation of the difference data compared to the initial and final zero data shows there is no common variation in the two sets of zero readings.

Figure 4 shows the corrected power for the last step in the cycle using the two different subtraction methods. The black trace shows the data when the initial zero reading is subtracted, and the red trace shows the value obtained when the zero is subtracted using a linear interpolation between the initial and final zero measurements. The subtraction method does not appear to significantly influence the magnitude of the fluctuations, which is consistent with the conclusion in the previous paragraph that there is no common variation between the initial and final zero readings.

Table 2 lists the average power readings, the standard deviations, and twice the relative standard deviations for all four levels with the offset subtracted by using a) the initial zero reading and b) a linear interpolation of the initial and final zero readings for 500 cycles. Figure 4 and Table 2 demonstrate that 1) a linear interpolation of the initial and final zero readings does not significantly improve the measurement uncertainty and b) higher fluctuations are observed in the environmental chamber compared 
to the measurements in open air (see Table 1). Because the chamber uses a fan to produce uniform temperatures by rapid air movement, the wind turbulence and perhaps also the acoustic noise and vibration of the operating environmental chamber affect the power meter stability. Although the large cyclic variations due to the changes in temperature are absent, larger changes between the initial and final zero readings are now limiting the measurement.

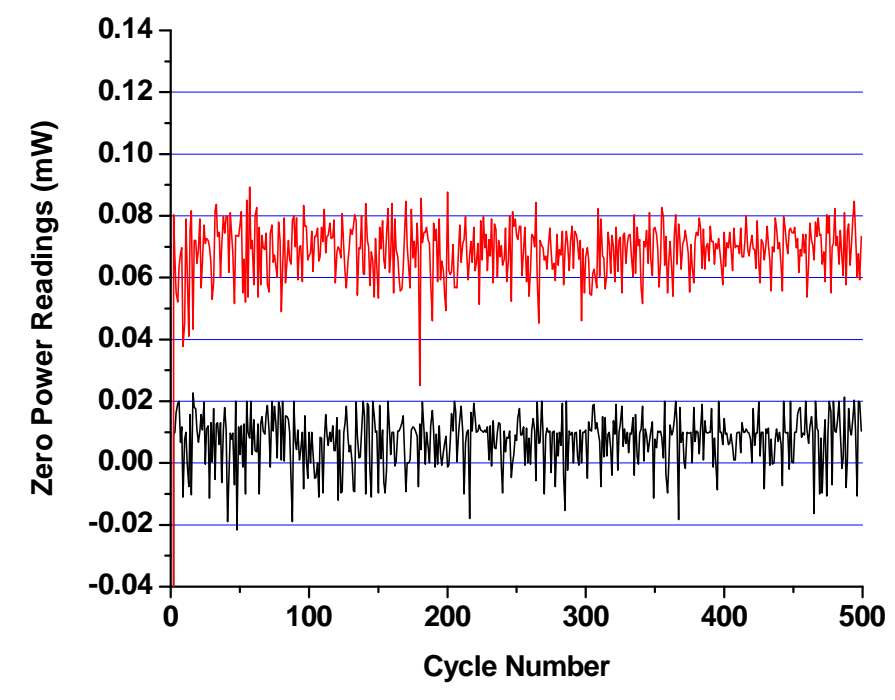

Figure 3. Initial (black) and Final (red) Zero Readings from the Power Meter in the Environmental Chamber at $30.0^{\circ} \mathrm{C}$

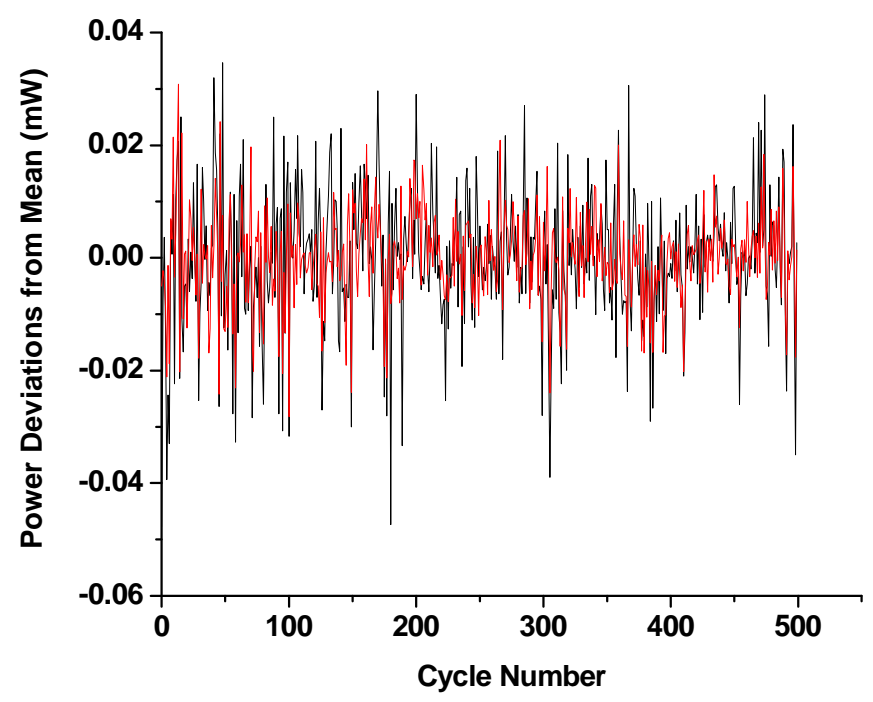

Figure 4. Power Deviations from the Mean Using Initial Zero Subtraction (black) and a Linear Interpolation Zero Subtraction (red) for the Measurements in the Environmental Chamber at $30.0^{\circ} \mathrm{C}$ 
Table 2. Power and Relative Confidence Limits of Power Meter Head in Operating Environmental Chamber at $30.0^{\circ} \mathrm{C}$ for 500 Cycles Using Both a) an Initial Zero Subtraction and b) a Linear Interpolation

\begin{tabular}{||l|c|c|c|c||}
\hline \multicolumn{5}{|c||}{ a) Initial Zero Subtraction } \\
\hline Average Power $(\mathrm{mW})$ & 1.129 & 2.263 & 3.377 & 4.447 \\
\hline Standard Deviation $(\mu \mathrm{W})$ & 8.7 & 10.3 & 10.7 & 12.2 \\
\hline $2 \times$ Rel. Std. Dev. & $1.55 \%$ & $0.91 \%$ & $0.64 \%$ & $0.55 \%$ \\
\hline \multicolumn{6}{||c|}{ b) Linear Interpolation Zero Subtraction } \\
\hline Average Power $(\mathrm{mW})$ & 1.116 & 2.238 & 3.340 & 4.397 \\
\hline Standard Deviation $(\mu \mathrm{W})$ & 7.8 & 8.5 & 8.1 & 8.1 \\
\hline $2 \times$ Rel. Std. Dev. & $1.39 \%$ & $0.76 \%$ & $0.49 \%$ & $0.37 \%$ \\
\hline \hline
\end{tabular}

\subsection{Idle Environmental Chamber}

Since we believed the wind and vibration of the active temperature regulation system in the environmental chamber was responsible for the increased variation on the power meter, we cooled the environmental chamber to near room temperature and then shut it off expecting better performance. Figure 5 shows the power meter zero measurements for almost 500 cycles in the idle environmental chamber. The mean of the initial zero readings is $-16.7 \mu \mathrm{W} \pm 4.6 \mu \mathrm{W}$ whereas the mean of the final zero readings is $55.1 \mu \mathrm{W} \pm 4.6 \mu \mathrm{W}$. Subtraction of the initial and final zero readings yields a mean signal of $71.9 \mu \mathrm{W} \pm 2.1 \mu \mathrm{W}$. The standard deviation for the initial zero readings is lower than the two cases presented above indicating the fluctuations are minimized. Furthermore, subtraction of the initial and final zero readings has a smaller standard deviation; therefore, the fluctuations between the initial and final zero readings is much lower for this scenario because fewer changes are occurring during the measurement cycle.

Figure 6 shows the corrected power for the last step in the cycle using the two different subtraction methods. The black trace shows the data when the initial zero reading is subtracted, and the red trace shows the values obtained when the zero is subtracted using a linear interpolation between the initial and final zero measurements. Similar to the operating environmental chamber, the subtraction method does not have a significant impact on most of the fluctuations. In this case, however, it is because the variations during the measurement cycle have been drastically reduced.

Table 3 lists the average power, standard deviation, and twice the relative standard deviation of these power meter readings with subtraction of a) the initial zero readings and b) linear interpolation of the initial and final zero readings for the power measurements. The standard deviations are about half of what was measured in the open air in the lab except for the lowest power level. Thus, effects from the air currents and temperature cycling from the HVAC system are reduced in the closed environmental chamber compared to open air. For these measurements, the temperature is not changing during the course of a measurement cycle so that the uncertainty is the same for both subtraction methods. An offset between the initial and final zero readings is still present due to the local heating of the power meter, which changes the absolute value of the average power as indicated in Table 3. 


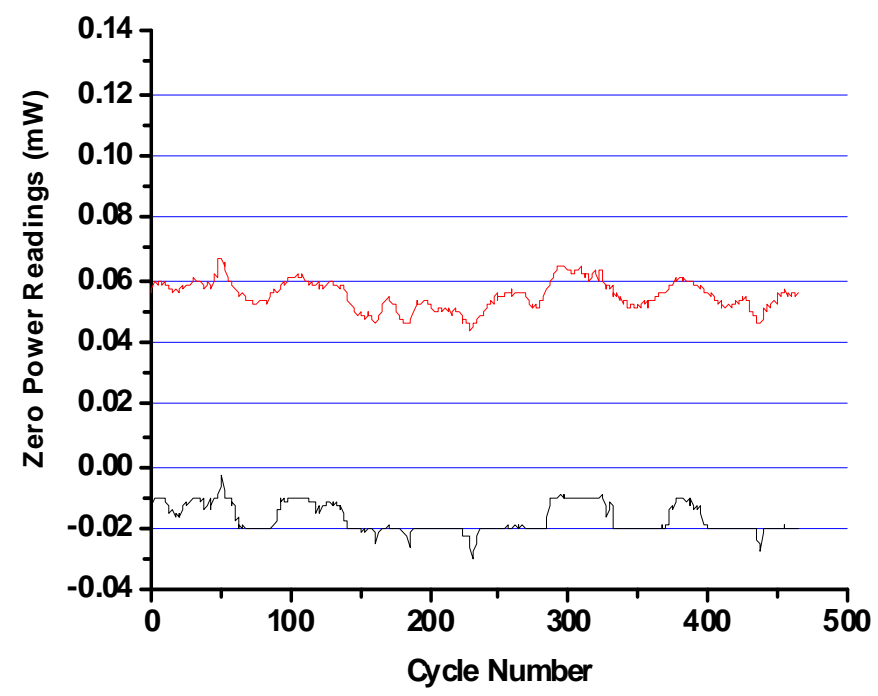

Figure 5. Initial (black) and Final (red) Zero Power Readings from the Power Meter in the Idle Environmental Chamber

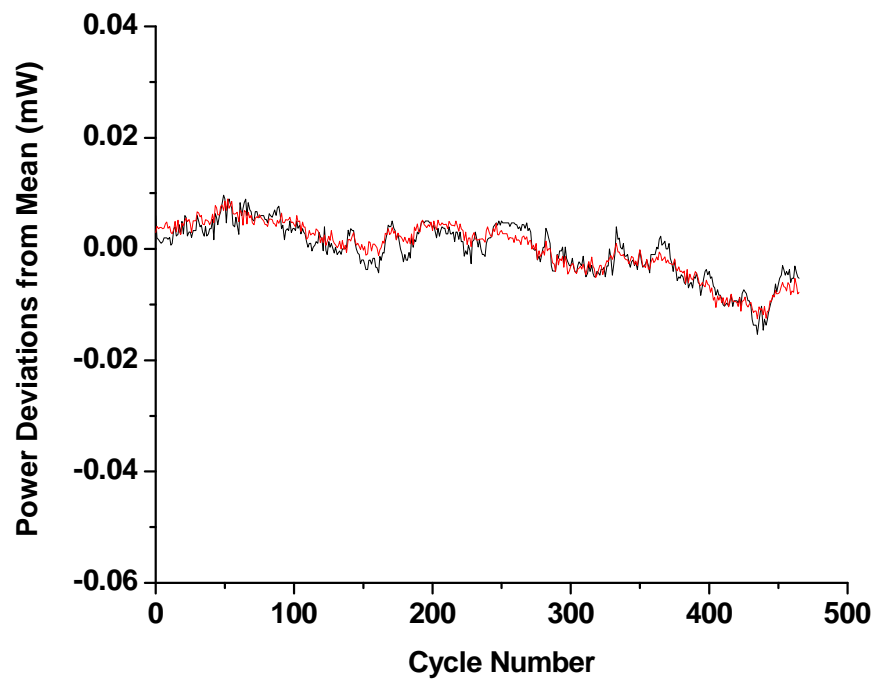

Figure 6. Power Deviations from the Mean Using Initial Zero Subtraction (black) and a Linear Interpolation Zero Subtraction (red) for the Measurements in an Idle Environmental Chamber

We left the system collecting data to get a long data set. Concatenating all the data taken with the idle environmental chamber provided 2172 cycles (over 2 weeks of data). The mean of the initial zero readings is $22.4 \mu \mathrm{W} \pm 28.1 \mu \mathrm{W}$ whereas the mean of the final zero readings is92.4 $\mu \mathrm{W} \pm 26.4$. Subtraction of the initial and final zero readings yields a mean signal of $70.0 \mu \mathrm{W} \pm 2.7 \mu \mathrm{W}$. The factor of 10 reduction in standard deviation due to the subtraction indicates a common source for the majority of the variations. 
Table 3. Power and Relative Confidence Limits of Power Meter Head in Idle Environmental Chamber for 472 Cycles Using Both a) an Initial Zero Subtraction and b) a Linear Interpolation

\begin{tabular}{||l|c|c|c|c||}
\hline \hline \multicolumn{5}{|c||}{ a) Initial Zero Subtraction } \\
\hline Average Power $(\mathrm{mW})$ & 1.136 & 2.275 & 3.386 & 4.445 \\
\hline Standard Deviation $(\mu \mathrm{W})$ & 3.0 & 3.0 & 4.4 & 4.9 \\
\hline $2 \times$ Rel. Std. Dev. & $0.52 \%$ & $0.27 \%$ & $0.26 \%$ & $0.22 \%$ \\
\hline \multicolumn{6}{||c|}{ b) Linear Interpolation Zero Subtraction } \\
\hline Average Power $(\mathrm{mW})$ & 1.112 & 2.247 & 3.343 & 4.387 \\
\hline Standard Deviation $(\mu \mathrm{W})$ & 2.8 & 2.7 & 4.4 & 4.9 \\
\hline $2 \times$ Rel. Std. Dev. & $0.50 \%$ & $0.24 \%$ & $0.26 \%$ & $0.22 \%$ \\
\hline \hline
\end{tabular}

Figure 7 shows the deviations of the power readings (after subtracting initial zero readings) of the concatenated data set. There is clearly a small common downward drift of all four sets of power readings indicating a possible common cause.

Table 4 lists the average power, standard deviation, and twice the relative standard deviation of these power meter readings with subtraction of a) the initial zero readings and b) linear interpolation of the initial and final zero readings. Similar results to those in Table 3 are observed for the longer time period except greater variation is obtained in the measurements due to the initial drift in power seen in Figure 7. This drift may result from a temperature equilibration within the environmental chamber.

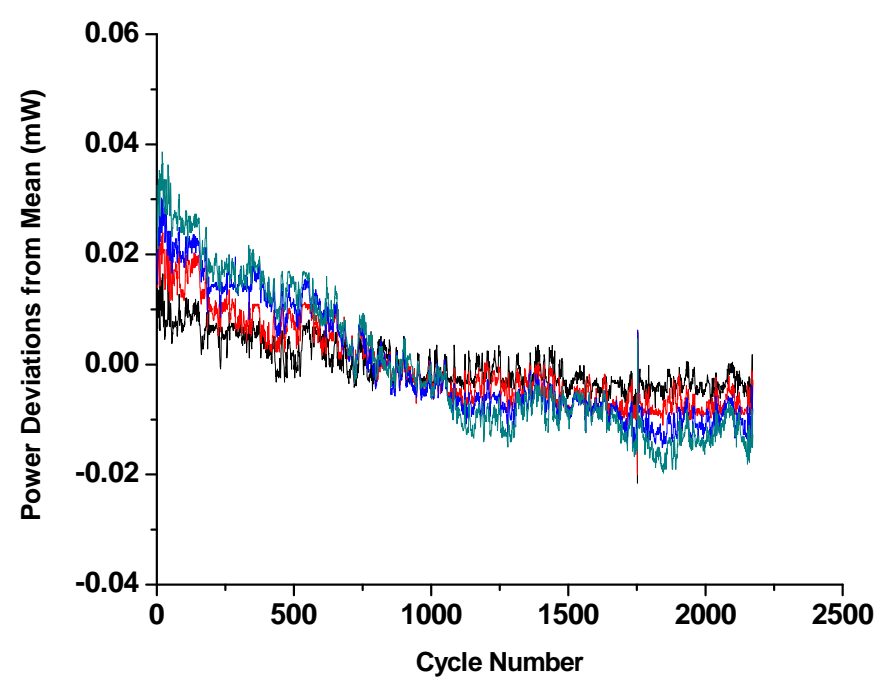

Figure 7. Power Deviations from the Mean Using Initial Zero Subtraction for the Measurements in the Idle Environmental Chamber. The black, red, blue, and dark cyan traces show the data for the first through the fourth steps, respectively. 
Table 4. Power and Relative Confidence Limits of Power Meter Head in Idle Environmental Chamber for 2172 Cycles Using Both a) an Initial Zero Subtraction and b) a Linear Interpolation

\begin{tabular}{||l|c|c|c|c||}
\hline \multicolumn{5}{|c||}{ a) Initial Zero Subtraction } \\
\hline Average Power $(\mathrm{mW})$ & 1.133 & 2.269 & 3.376 & 4.443 \\
\hline Standard Deviation $(\mu \mathrm{W})$ & 4.4 & 7.9 & 10.8 & 13.2 \\
\hline $2 \times$ Rel. Std. Dev. & $0.78 \%$ & $0.70 \%$ & $0.64 \%$ & $0.60 \%$ \\
\hline \multicolumn{6}{|c|}{ b) Linear Interpolation Zero Subtraction } \\
\hline Average Power $(\mathrm{mW})$ & 1.119 & 2.241 & 3.334 & 4.377 \\
\hline Standard Deviation $(\mu \mathrm{W})$ & 4.4 & 8.0 & 11.0 & 13.6 \\
\hline $2 \times$ Rel. Std. Dev. & $0.79 \%$ & $0.71 \%$ & $0.66 \%$ & $0.62 \%$ \\
\hline
\end{tabular}

\subsection{Inexpensive Custom Environmental Enclosure}

Because the environmental chamber did not improve the stability measurements when it was in operation, we designed an inexpensive environmental enclosure for long-term QCL stability experiments that does not use fans to regulate the temperature. We arranged a simple plastic tub inside a Styrofoam cooler to provide insulated housing. To remove the waste heat from the QCL and the thermo-electric cooler (TEC), we modified an ILX temperature-controlled mount for c-mounted lasers for water cooling instead of fan cooling. This modification involved milling off the air cooling fins and attaching a milled aluminum block with water passages. A circulator is used to provide the water cooling in which holes were drilled into the plastic tub for the water cooling lines. Figure 8 shows a photograph of the set-up.

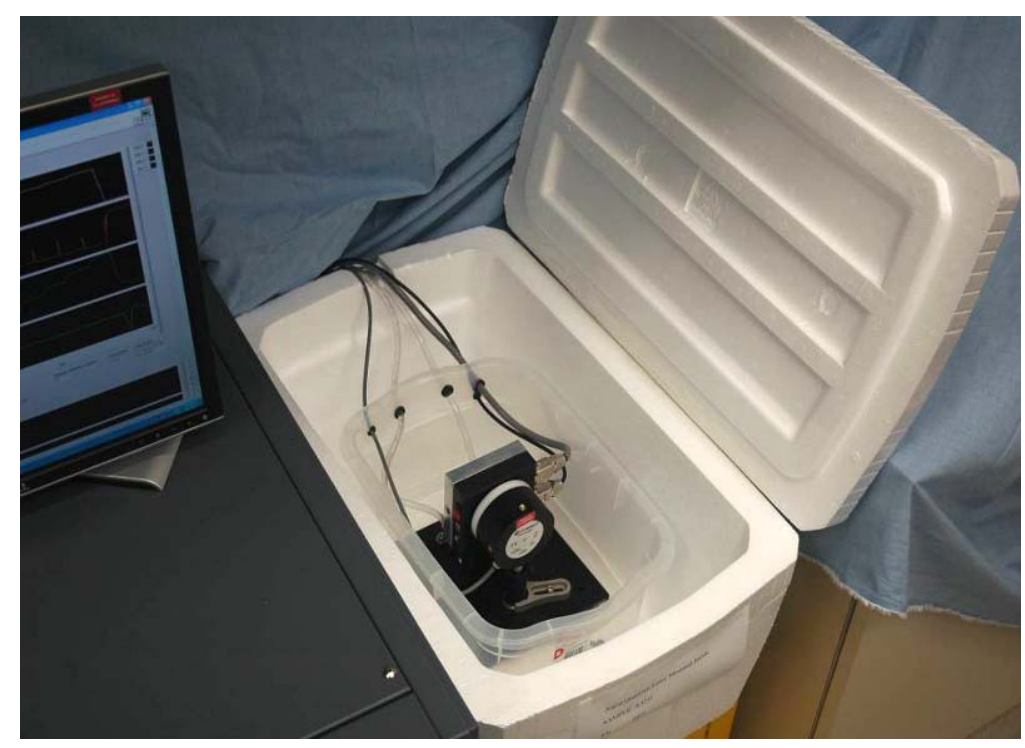

Figure 8. Simple Environmental Enclosure for Stability Testing 


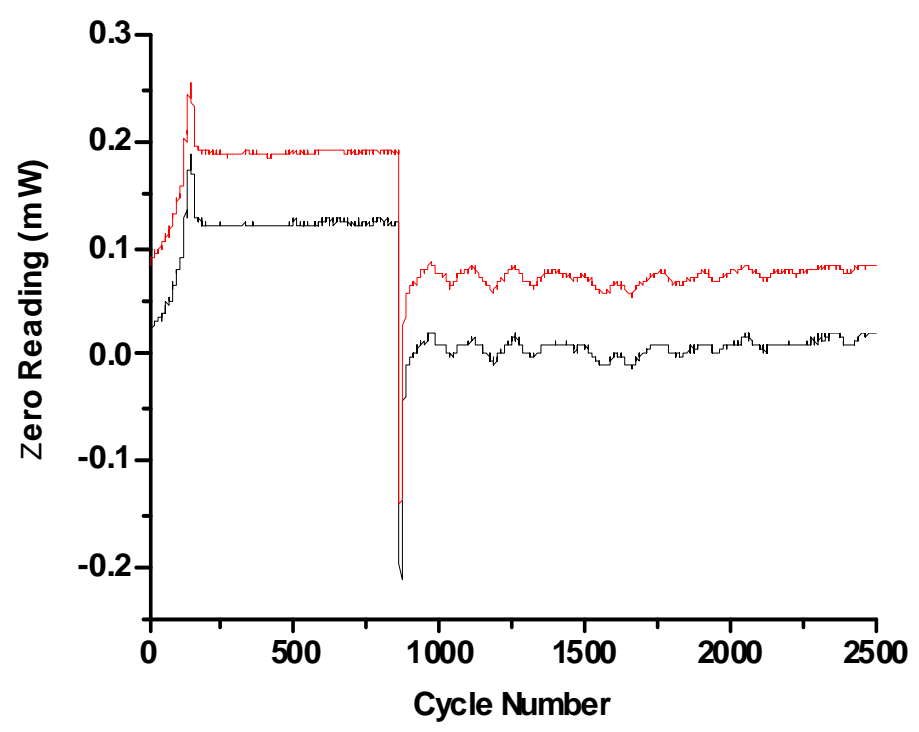

Figure 9. Initial (black) and Final (red) Zero Power Readings from the Power Meter in the Custom Environmental Chamber

We collected data in this new setup with the circulating tap water controlled to $22.0^{\circ} \mathrm{C}$ using the resistor that is attached to the power meter to provide a thermal signal. Figure 9 shows the initial and final zero readings for the power meter for data collected for 2525 cycles for over 500 hours of operation. The sharp drop in the zero readings in Figure 9 at the 864th cycle (around 144 hours) corresponds to water being added to the water circulator because the level was well below the recommended minimum level. Before adding water, fluctuations between the initial and final zero readings are approximately $2 \mu \mathrm{W}$, similar to the results in the idle environmental chamber. Thus, this custom chamber provides sufficient stability for the measurements.

Figure 10 shows the variations about the means of the corrected power readings using the interpolated zero subtraction technique. The spike in the figure corresponds to the rapid change in the zero reading that occurred when water was added to the circulator.

Table 5 lists the means, standard deviations, and twice the relative standard deviations for the data in Figure 10. These standard deviations are roughly a factor of 2 smaller than those for the data shown in Table 4 with a similar number of cycles.

This data, which was collected for over 500 hours, demonstrates very good measurements can be obtained in this custom environmental chamber. The average uncertainty of the power meter is about $3 \mu \mathrm{W}$. 


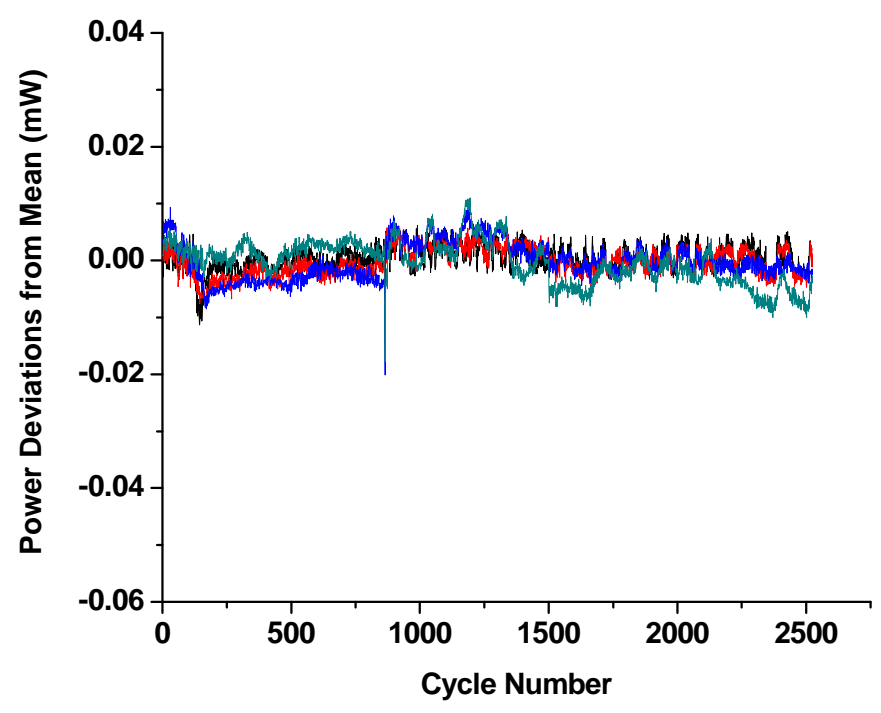

Figure 10. Power Deviations from the Mean Using an Interpolated Zero Subtraction for the Measurements in the Custom Environmental Chamber. The black, red, blue, and dark cyan traces show the data for the first through the fourth steps.

Table 5. Power and Relative Confidence Limits of Power Meter Head in Custom Environmental Chamber for 2525 Cycles Using Both a) an Initial Zero Subtraction and b) a Linear Interpolation

\begin{tabular}{||l|c|c|c|l||}
\hline \multicolumn{5}{|c||}{ a) Initial Zero Subtraction } \\
\hline Average Power $(\mathrm{mW})$ & 1.124 & 2.246 & 3.333 & 4.372 \\
\hline Standard Deviation $(\mu \mathrm{W})$ & 2.7 & 3.2 & 4.2 & 5.0 \\
\hline $2 \times$ Rel. Std. Dev. & $0.45 \%$ & $0.25 \%$ & $0.21 \%$ & $0.19 \%$ \\
\hline \multicolumn{6}{||c|}{ b) Linear Interpolation Zero Subtraction } \\
\hline Average Power $(\mathrm{mW})$ & 1.110 & 2.219 & 3.293 & 4.319 \\
\hline Standard Deviation $(\mu \mathrm{W})$ & 2.3 & 2.5 & 3.3 & 3.7 \\
\hline $2 \times$ Rel. Std. Dev. & $0.41 \%$ & $0.23 \%$ & $0.20 \%$ & $0.17 \%$ \\
\hline \hline
\end{tabular}

\subsection{3-mW Scale and TEC Running in Custom Environmental Enclosure}

An ILX temperature controller was installed to actively control the temperature of the laser mount. We tested the effect of a better readout resolution by operating the power meter on the 3-mW scale versus the $30-\mathrm{mW}$ scale. On this scale, the readout resolution is $0.001 \mathrm{~mW}$. The ILX was controlling the laser mount to $17.000^{\circ} \mathrm{C}$, and the voltages applied to the resistor were reduced to keep the power meter readings below $3 \mathrm{~mW}$. Figure 11 shows the data from the initial and final zero readings where many minor features are present in both traces. This data was collected for 240 cycles or over 40 hours. 


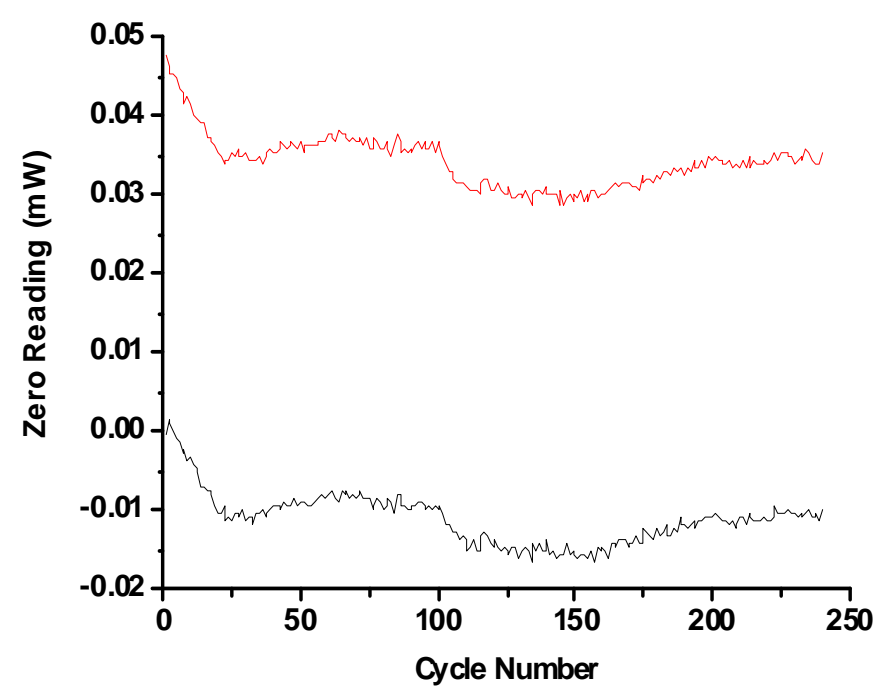

Figure 11. Initial (black) and Final (red) Zero Power Readings from the Power Meter in the Custom Environmental Chamber with a Resolution of $0.001 \mathrm{~mW}$

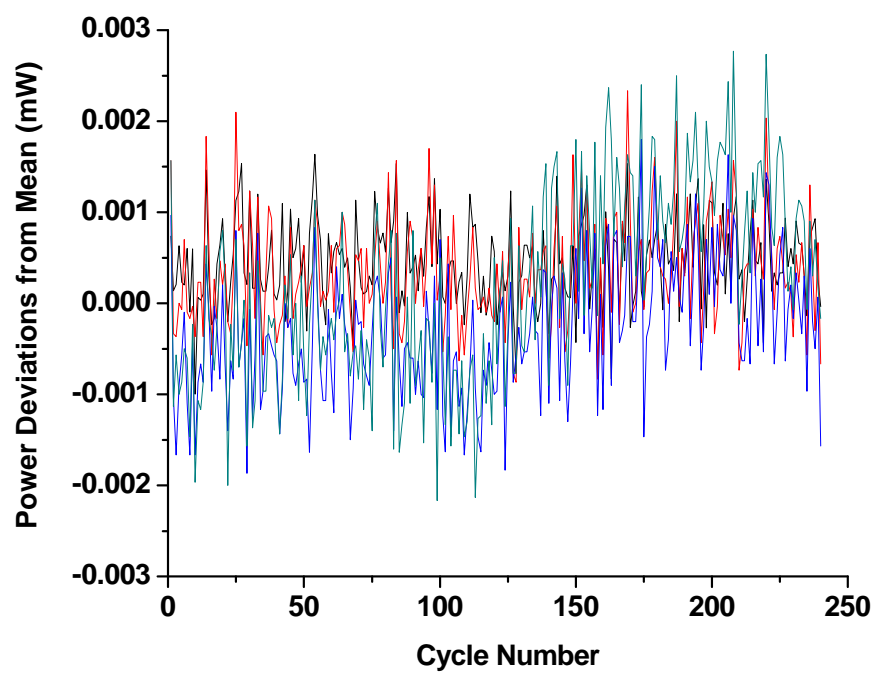

Figure 12. Power Deviations from the Mean Using Initial Zero Subtraction for the Measurements in the Custom Environmental Chamber. The black, red, blue, and dark cyan traces show the data for the first through the fourth steps above threshold, respectively.

Figure 12 shows the variations from the means of the zero subtracted power readings using just the initial zero reading for subtraction. 
Table 6. Power and Relative Confidence Limits of Power Meter Head in Custom Environmental Enclosure for 240 Cycles Using an Initial Zero Subtraction and the 3-mW Scale on the Power Meter

\begin{tabular}{|l|l|l|l|l|}
\hline Average Power $(\mathrm{mW})$ & 0.756 & 1.521 & 2.255 & 2.940 \\
\hline Standard Deviation $(\mu \mathrm{W})$ & 0.48 & 0.59 & 0.73 & 1.1 \\
\hline $2 \times$ Rel. Std. Dev. & $0.13 \%$ & $0.08 \%$ & $0.06 \%$ & $0.07 \%$ \\
\hline
\end{tabular}

Table 6 lists the results for the means, standard deviations, and twice the relative standard deviations. Better readout resolution on the power meter clearly gives better zero subtraction. Unfortunately, for most of our systems, we do not want to limit the power below $3 \mathrm{~mW}$ so that the measurement system will have the higher uncertainty. To limit its effect, we can measure the laser at higher output powers so that the effect is not as dramatic on the measurement stability.

\subsection{Stability of QCL in Custom Environmental Enclosure}

\subsection{Below Threshold for cw RT QCL}

A cw RT QCL was mounted in the modified mount in the custom environmental enclosure. The temperature of the laser mount was controlled at $20.000^{\circ} \mathrm{C}$ with the power meter on the 30 -mW scale. The stability of the power meter was further tested by measuring the background signal on the power meter by choosing all current levels below threshold. The scheme provided six current levels all below threshold followed by several 1-second steps to ramp the current down to zero. For each step (except for the steps ramping down the current), the current is held constant for 15 seconds and after a 10 -second delay, the data is averaged for 5 seconds. A new cycle begins every 10 minutes so that there are 6 cycles per hour. Figure 13 shows the power meter readings for all of the levels. The stepwise structure reflects the $0.01-\mathrm{mW}$ output resolution of the power meter.

The first step is used to provide an initial zero reading that is subtracted from the power readings for the next five steps. Figure 14 shows the zero subtracted power meter readings at the five current levels that are all below threshold. The shifts in the power readings are either due to the changes in heat from the QCL and mount that is measured by the power meter or due to heating of the absorbing medium of the thermopile which changes the offset.

Table 7 shows the averages and standard deviations of this data. The increases in power meter readings are linear with the current setting for the steps within $1 \%$. This effect is consistent with the reading tracking the change in heat deposited in the QCL.

The average standard deviation is $2.8 \mu \mathrm{W}$, which agrees well with the values shown in Table 5. This standard deviation will be used as the standard error for the measurement due to the power meter. 


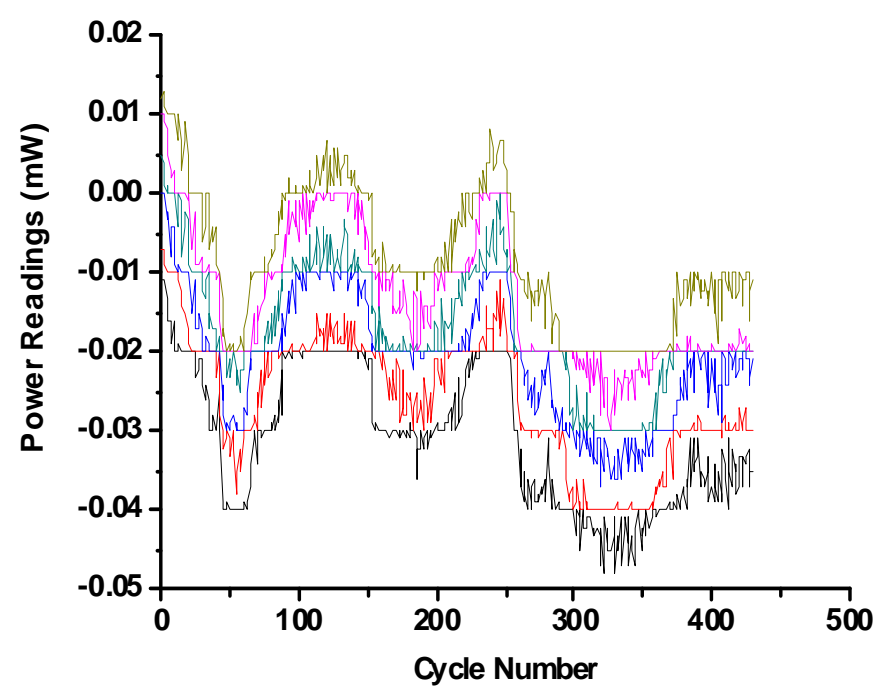

Figure 13. Power Meter Readings for All Six Steps Below Threshold in the Custom Environmental Chamber. The black, red, blue, dark cyan, magenta, and dark yellow traces represent the first through the sixth steps, respectively.

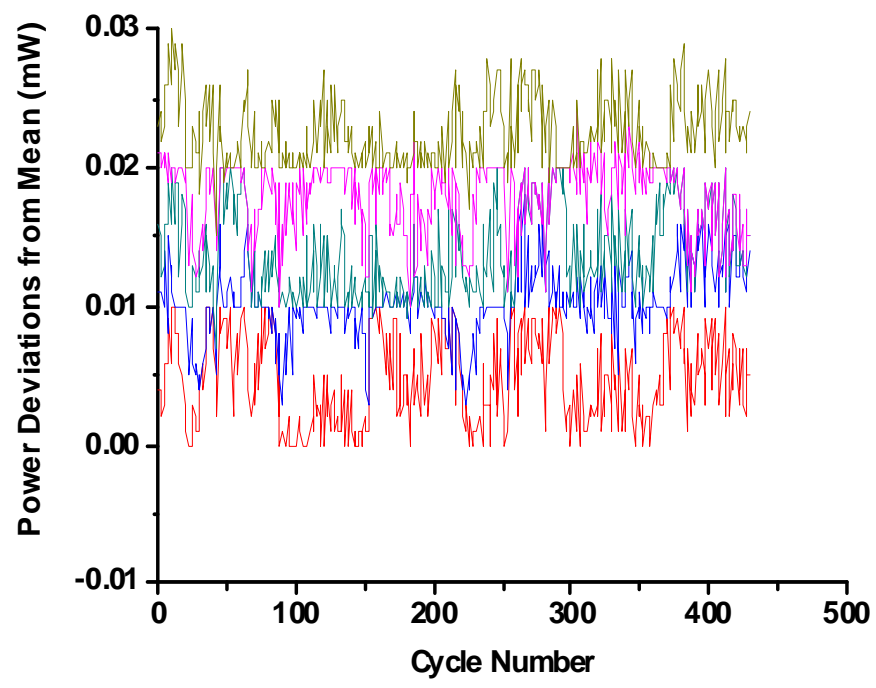

Figure 14. Power Deviations from the Mean Using Initial Zero Subtraction for the Five Steps at Different Currents, which are All below Threshold. The red, blue, dark cyan, magenta, and dark yellow traces represent the second through the sixth steps, respectively. 
Table 7. Zero Subtracted Power Meter Readings and Standard Deviations for Five Different Current Levels Applied to a QCL in a Custom Environmental Enclosure Along with the Power Meter Head over 429 Cycles. The current levels for the QCL were all below threshold, and the power meter was set to the $30 \mathrm{~mW}$ scale.

\begin{tabular}{|l|l|l|l|l|l|}
\hline Average Power $(\mathrm{mW})$ & 0.005 & 0.010 & 0.014 & 0.018 & 0.022 \\
\hline Standard Deviation $(\mu \mathrm{W})$ & 3.2 & 2.6 & 3.2 & 2.6 & 2.5 \\
\hline
\end{tabular}

\subsection{Above Threshold for cw RT QCL}

A scheme that provided current levels for a pre-heat (power meter reading R0), a sub-threshold power meter zero (power meter reading R1), five above threshold currents (power meter reading R2-R6), followed by several one-second steps to ramp the current down to zero was selected. For each step (except for the current ramp down steps), the current is held constant for 15 seconds and after a 10-second delay, the data is averaged for the remaining 5 seconds. The main part of the cycle lasts for 105 seconds so that only an initial zero reading is used to adjust for the offset on the power meter because fluctuations should be minimized, making a linear interpolation unnecessary. A new cycle begins every 10 minutes. Figure 15 shows the zero subtracted power readings for the five levels over about 27 hours with the temperature for the laser mount controlled to $17.000^{\circ} \mathrm{C}$. The results in Figure 15 are significantly worse than the power meter by itself shown in Figure 10 as well as the results below threshold shown in Figure 14.

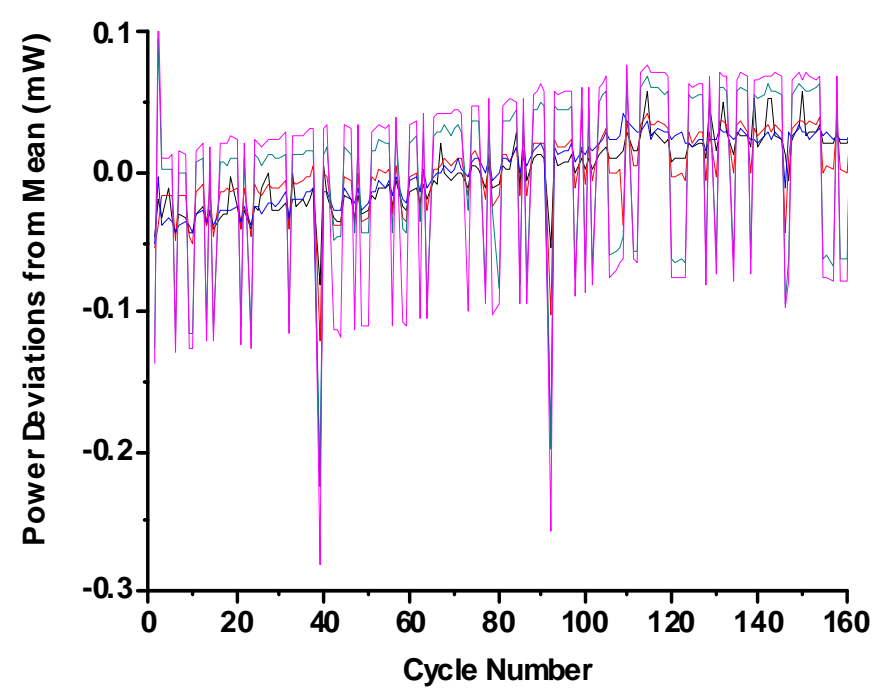

Figure 15. Power Deviations from the Mean Using Initial Zero Subtraction at Five Different Current Levels for a QC Laser that is Operated cw in the Custom Environmental Chamber. The black, red, blue, dark cyan, and magenta traces represent the first through the fifth steps above threshold, respectively. 
Table 8 lists the average powers, standard deviations, and twice the relative standard deviations of the data in Figure 15.

The variation in the above threshold power measurements appears to jump among a set of preferred values. Figure 16 shows an expanded section of Figure 15, where a strong correlation between the variations in the power levels for a given cycle is apparent. A possible source of these variations is the set of modes in which the QCL starts lasing for each cycle. These modes would be determined by the probabilities of the population of thermal photons and spontaneous emission photons in the QCL waveguide that build up above threshold. This hypothesis could be tested by changing from a DC current to a $50 \%$ duty cycle, $40 \mathrm{kHz}, 100 \%$ modulation depth drive current waveform. This modulated waveform would give a random starting point for lasing for each cycle of the current waveform. Another possibility is some type of temperature-driven instability that might be reduced by better temperature control or perhaps changing the distance between the front of the power meter head and the cold face of the ILX laser mount.

Table 8. Zero Subtracted Power Meter Readings and Standard Deviations for Five Different DC Current Waveforms Applied to a QCL in the Custom Enclosure for 160 Cycles

\begin{tabular}{||l|c|c|c|c|c||}
\hline \hline Average Power $(\mathrm{mW})$ & 5.264 & 11.715 & 17.301 & 22.945 & 28.629 \\
\hline Standard Deviation $(\mu \mathrm{W})$ & 25.4 & 27.0 & 33.1 & 59.0 & 75.7 \\
\hline $2 \times$ Rel. Std. Dev. & $1.0 \%$ & $0.5 \%$ & $0.4 \%$ & $0.5 \%$ & $0.5 \%$ \\
\hline \hline
\end{tabular}

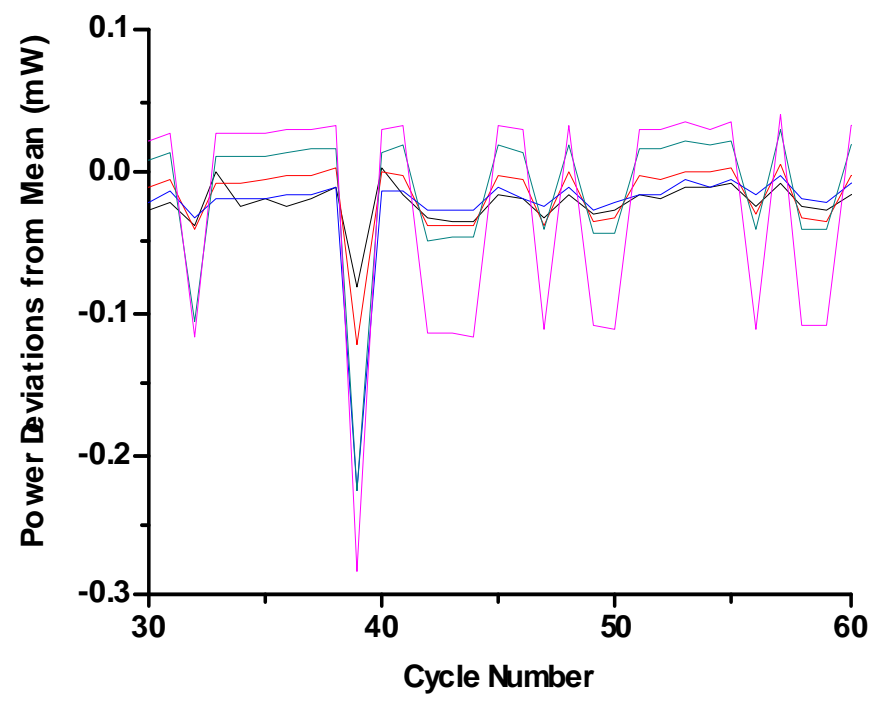

Figure 16. Expanded View of Variations in Power Meter Readings from Their Means for the Five Above Threshold Steps Shown in Figure 15. The strong correlations in the variations between steps in a given cycle are striking. 


\subsection{Above Threshold for QCL with Modulated Currents}

The experiments were repeated with a laser-mount temperature of $20.000^{\circ} \mathrm{C}$ and similar results are observed in which the power varies in discrete steps indicating a different set of longitudinal modes with lasing when the laser is operated cw. The correlations in the power deviations between steps in the same cycle suggest that the power at a given current is influenced by the set of longitudinal modes that first started lasing. To test this hypothesis, we modified the LabVIEW program to have the output from the digital-to-analog converter that controls the QCL current supply to be a square wave with $50 \%$ duty cycle between $0 \mathrm{~V}$ and Vpeak. A frequency of $40 \mathrm{kHz}$ was selected for the current modulation.

Figure 17 shows the deviations of the zero subtracted power meter readings from the means over the initial 65 hours (cycle period is 10 minutes). Table 9 lists the means, standard deviations, and twice the relative standard deviations of the data shown in Figure 17 as well as for the peak currents measured from the monitor output. These variations in the current may reflect the imperfect averaging out of the $40 \mathrm{kHz}$ modulation of the current because based on the light versus current (L-I) curve, a current variation of $0.1 \mathrm{~mA}$ would give a power variation of $0.041 \mathrm{~mW}$, which does not correspond to the observed power variations of $0.004 \mathrm{~mW}$. The current modulation, however, has substantially reduced the variations in the power readings compared to using DC current steps.

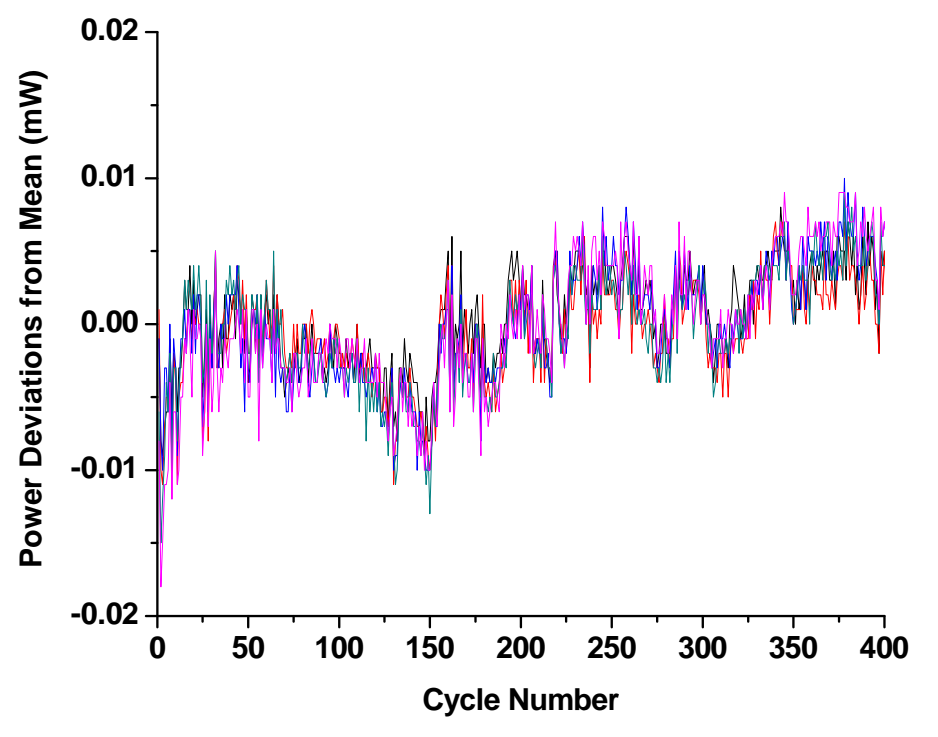

Figure 17. Power Deviations from the Mean Using Initial Zero Subtraction at Five Different Current Levels for a QC Laser Using a 40 kHz, 50\% Duty Cycle Current Modulation in the Custom Environmental Chamber. The black, red, blue, dark cyan, and magenta traces represent the first through the fifth steps above threshold, respectively. 
Table 9. Zero Subtracted Power Meter Readings and Standard Deviations for Five Different Modulated Current Waveforms Applied to a QCL in a Custom Environmental Enclosure. The average peak currents for the steps and their standard deviations is also provided.

\begin{tabular}{||l|c|c|c|c|c||}
\hline \hline Average Power $(\mathrm{mW})$ & 6.046 & 11.864 & 17.513 & 23.076 & 28.681 \\
\hline Standard Deviation $(\mu \mathrm{W})$ & 3.4 & 3.4 & 4.1 & 4.0 & 4.7 \\
\hline $2 \times$ Rel. Std. Dev. & $0.11 \%$ & $0.06 \%$ & $0.05 \%$ & $0.03 \%$ & $0.03 \%$ \\
\hline Average Power $(\mathrm{mW})$ & -267.07 & -282.14 & -296.20 & -309.53 & -322.55 \\
\hline Standard Deviation $(\mu \mathrm{W})$ & 0.10 & 0.12 & 0.11 & 0.11 & 0.12 \\
\hline $2 \times$ Rel. Std. Dev. & $0.08 \%$ & $0.09 \%$ & $0.08 \%$ & $0.07 \%$ & $0.07 \%$ \\
\hline
\end{tabular}

\subsubsection{Temperature Profiles}

Figure 18 shows the temperature profiles with DC current steps and with $40 \mathrm{kHz}$ modulated current steps. As expected from the almost 50\% decrease in average current to the QCL when going from DC to modulated current, the maximum temperature excursions are about half as large for the current modulation steps than for the cw steps. However, the smaller temperature excursions that accompany the beginning of each of the smaller current increases when the QCL is above threshold are about the same in both cases with a maximum deviation of about $0.005^{\circ} \mathrm{C}$. By the time power readings are collected for averaging, the temperature is $\pm 0.001^{\circ} \mathrm{C}$ of the set point of $20.000^{\circ} \mathrm{C}$.

\subsubsection{Long-term Measurements}

The experiments in Section 3.3.1 were continued to observe the long-term behavior. Figure 19 shows the data collected over 1500 cycles. The signal increases initially but appears to stabilize after about 150 hours of operation (around the $900^{\text {th }}$ cycle).

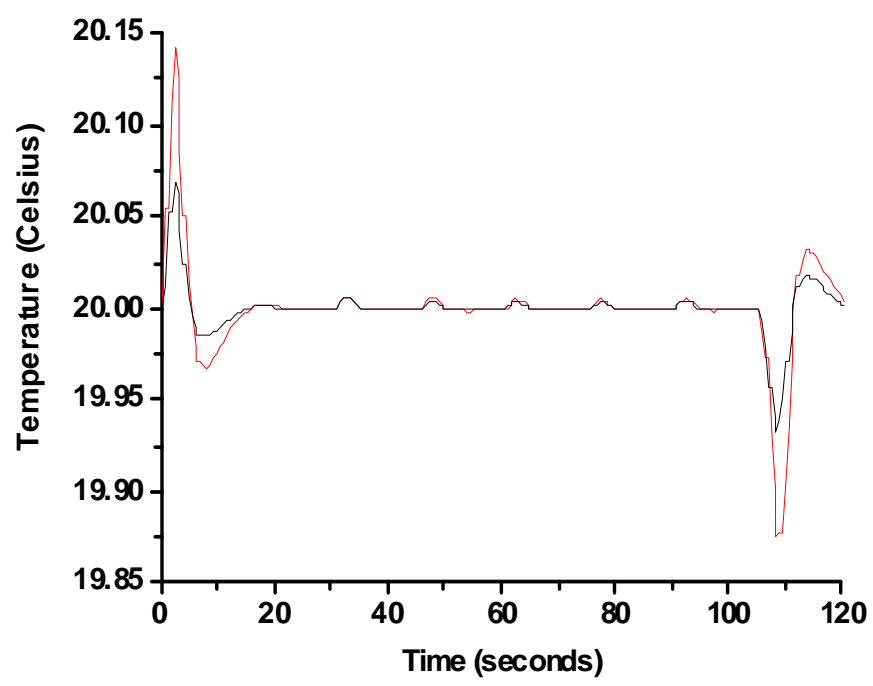

Figure 18. Temperature Profiles During a Single Cycle with DC Current Steps (red trace) and a $40 \mathrm{kHz}$ Modulated 50\% Duty Cycle Current Steps (black trace) 


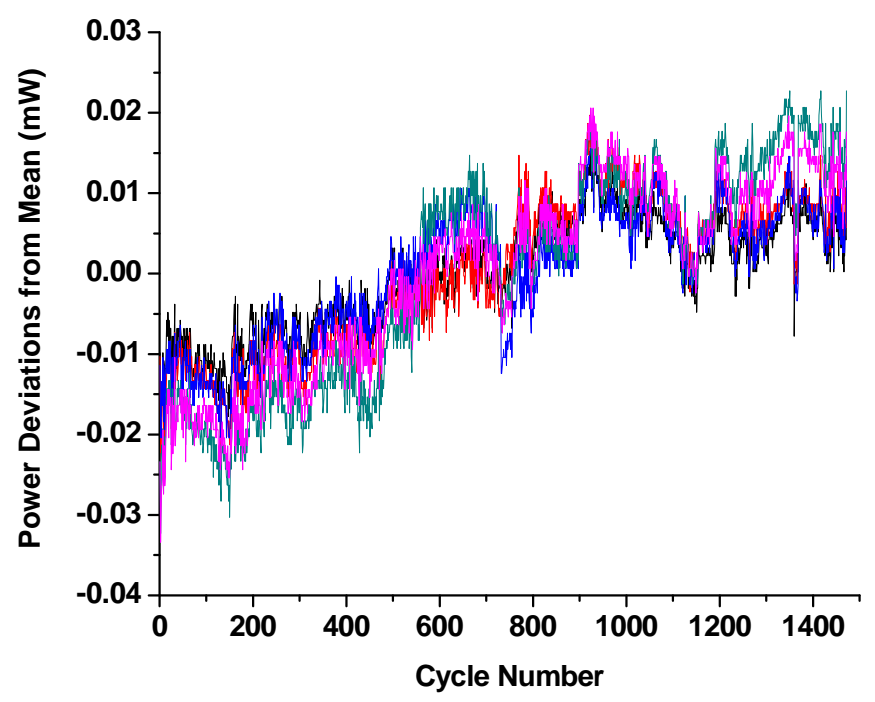

Figure 19. Power Deviations from the Mean Using Initial Zero Subtraction at Five Different Current Levels for a QC Laser Using a $40 \mathrm{kHz}, 50 \%$ Duty Cycle Current Modulation in the Custom Environmental Chamber. The black, red, blue, dark cyan, and magenta traces represent the first through the fifth steps above threshold, respectively.

Tables 10 and 11 list the means, standard deviations, and twice the relative standard deviations of the data shown in Figure 19. These tables list the standard deviation for the mean as well as the calculated standard deviation for the power meter (from Section 3.1). Based on propagation of errors, we can subtract out the error from the measurement to determine the fluctuations that predominantly result from the laser and the current supply, which is reported as the standard deviation for the laser. The variations are much smaller without the section with drift and may be partially limited by the 0.01-mW output resolution of the power meter output. For each step in each cycle, 10 power meter readings are averaged.

A significant fraction of the fluctuations can be attributed to the measurement. Thus, very low uncertainties $(<0.1 \%)$ have been achieved in this simple set-up. The increased fluctuations for the fourth and fifth steps may be attributed to the subtraction technique in which just the initial zero is used; therefore, larger fluctuations result for the last two steps because changes may have occurred during the measurement cycle.

Table 10. Zero Subtracted Power Meter Readings and Standard Deviations for the Data Shown in Figure 19

\begin{tabular}{|l|l|c|c|c|c||}
\hline Average Power $(\mathrm{mW})$ & 6.055 & 11.876 & 17.524 & 23.094 & 28.697 \\
\hline Standard Deviation $(\mu \mathrm{W})$ & 6.8 & 9.3 & 8.1 & 13.6 & 11.7 \\
\hline Std. Dev. from Power Meter $(\mu \mathrm{W})$ & 2.8 & 2.8 & 2.8 & 2.8 & 2.8 \\
\hline Std. Dev. for Laser $(\mu \mathrm{W})$ & 6.1 & 8.8 & 7.6 & 13.3 & 11.3 \\
\hline $2 \times$ Rel. Std. Dev. of Laser & $0.20 \%$ & $0.15 \%$ & $0.09 \%$ & $0.12 \%$ & $0.08 \%$ \\
\hline
\end{tabular}


Table 11. Zero Subtracted Power Meter Readings and Standard Deviations for the Data Shown in Figure 19 Excluding the First 900 Cycles. Statistics on data after 150 hours in Figure 19.

\begin{tabular}{||l|l|c|c|c|c||}
\hline \hline Average Power $(\mathrm{mW})$ & 6.060 & 11.883 & 17.530 & 23.106 & 28.707 \\
\hline Standard Deviation $(\mu \mathrm{W})$ & 3.6 & 3.7 & 3.6 & 5.0 & 4.5 \\
\hline Std. Dev. from Power Meter $(\mu \mathrm{W})$ & 2.8 & 2.8 & 2.8 & 2.8 & 2.8 \\
\hline Std. Dev. for Laser $(\mu \mathrm{W})$ & 2.2 & 2.4 & 2.2 & 4.1 & 3.5 \\
\hline $2 \times$ Rel. Std. Dev. of Laser & $0.07 \%$ & $0.04 \%$ & $0.02 \%$ & $0.03 \%$ & $0.02 \%$ \\
\hline
\end{tabular}

\subsection{Stability of QCL in Open Air with Modulated Currents}

We also started investigating the long-term stability of another laser system in FY06. This system is set up on an optical table in room air so that it is susceptible to the temperature fluctuations from the HVAC. Gray foam encloses the power meter to shield the detector from rapid changes such as personnel moving about the laboratory. The detector performance will be similar to the results in Section 2.1 for the open air measurements. The laser mount is stabilized to $15^{\circ} \mathrm{C}$. This system has five steps above threshold with each step lasting for 15 seconds. The total scheme lasts for 104 seconds with 20 seconds below threshold at the beginning of the scheme and 9 seconds below threshold as the system is ramped down. A lower duty cycle is used to minimize waste heat from the QCL because the mount is not fan- or water-cooled. The laser mount includes a fan for cooling, but it increased the noise on the measurement by a factor of two so its use was discontinued. This system is modulated at $100 \mathrm{kHz}$ with a $10 \%$ duty cycle so that the average power is lower than the system in the previous section. Over ten months, we have collected about 200,000 cycles in which a cycle is started every 134 seconds providing a 30 -second delay between cycles. Thus, 26 cycles per hour and 644 cycles per day are completed.

Since the data is collected in open air, larger fluctuations are observed with the zero power readings. Figure 20 shows the zero power readings for data taken over three weeks. Cyclic variations are observed due to the temperature fluctuations from the HVAC. The zero-subtracted power readings are shown in Figure 21 for all five steps.

Based on propagation of errors, we can subtract out the estimated error from the power meter to determine the fluctuations from the laser and its current supply. We use the values provided in Table 1 to estimate the uncertainty from the system. Table 12 lists the mean power, standard deviations for the mean, system and the laser, and twice the relative standard deviation for the laser for all five steps. For these measurements, the initial zero reading is used for subtracting the offset.

Thus, good results are obtained over a three-week time period in which the fluctuations were on the order of $3 \mu \mathrm{w}$; therefore, the laser power stability varied from about $1 \%$ for the lowest power level down to $0.1 \%$ for the highest power level.

We continued collecting data for this system over ten months. Figure 22 shows the data over this extended time period. The sharp spikes in the data mostly correspond to rapid changes in the zero reading. 


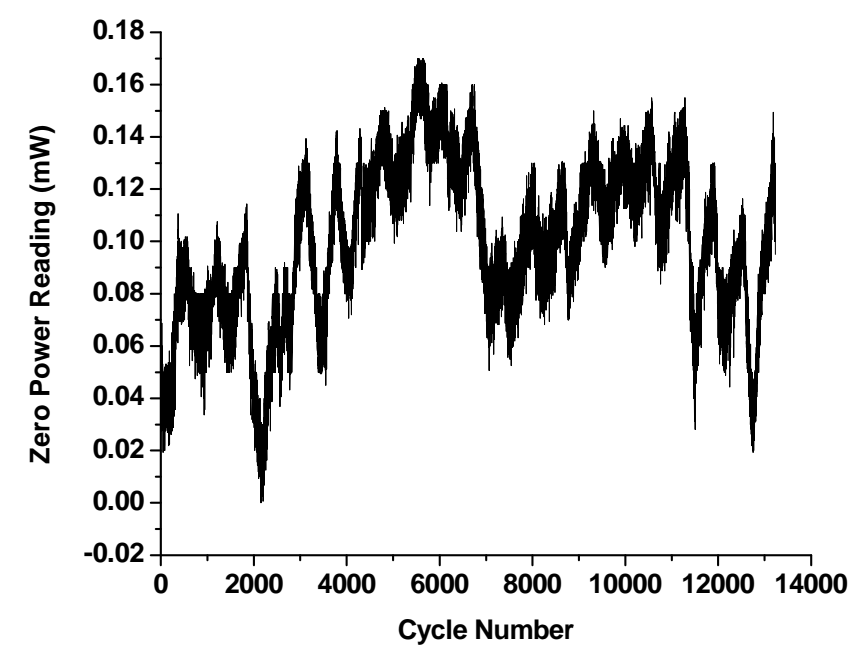

Figure 20. Initial Zero Power Readings from the Power Meter in the Open Air

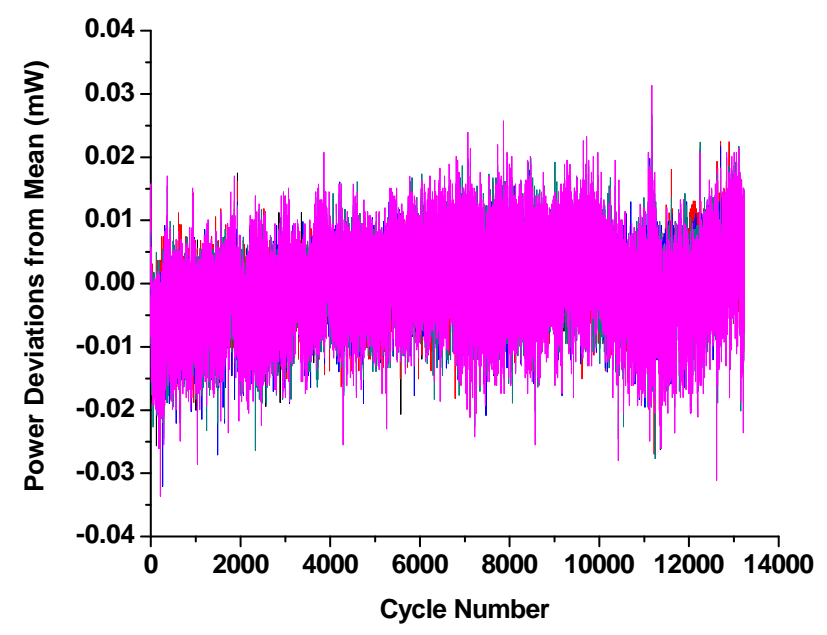

Figure 21. Power Deviations from the Mean Using Initial Zero Subtraction at Five Different Current Levels for a QC Laser Using a 100 kHz, 10\% Duty Cycle Current Modulation in Open Air Over Three Weeks. The black, red, blue, dark cyan, and magenta traces represent the first through the fifth steps above threshold, respectively.

Table 12. Zero Subtracted Power Meter Readings and Standard Deviations for the Data Shown in Figure 21

\begin{tabular}{||l|l|l|l|l|l||}
\hline Average Power $(\mathrm{mW})$ & 0.566 & 1.166 & 1.757 & 3.001 & 5.585 \\
\hline Standard Deviation $(\mu \mathrm{W})$ & 4.2 & 4.7 & 5.6 & 6.1 & 6.9 \\
\hline Std. Dev. from Power Meter $(\mu \mathrm{W})$ & 3.0 & 3.0 & 3.6 & 4.9 & 6.2 \\
\hline Std. Dev. for Laser $(\mu \mathrm{W})$ & 2.9 & 3.6 & 4.3 & 3.6 & 3.0 \\
\hline $2 \times$ Rel. Std. Dev. of Laser & $1.07 \%$ & $0.6 \%$ & $0.5 \%$ & 0.2 & 0.1 \\
\hline
\end{tabular}




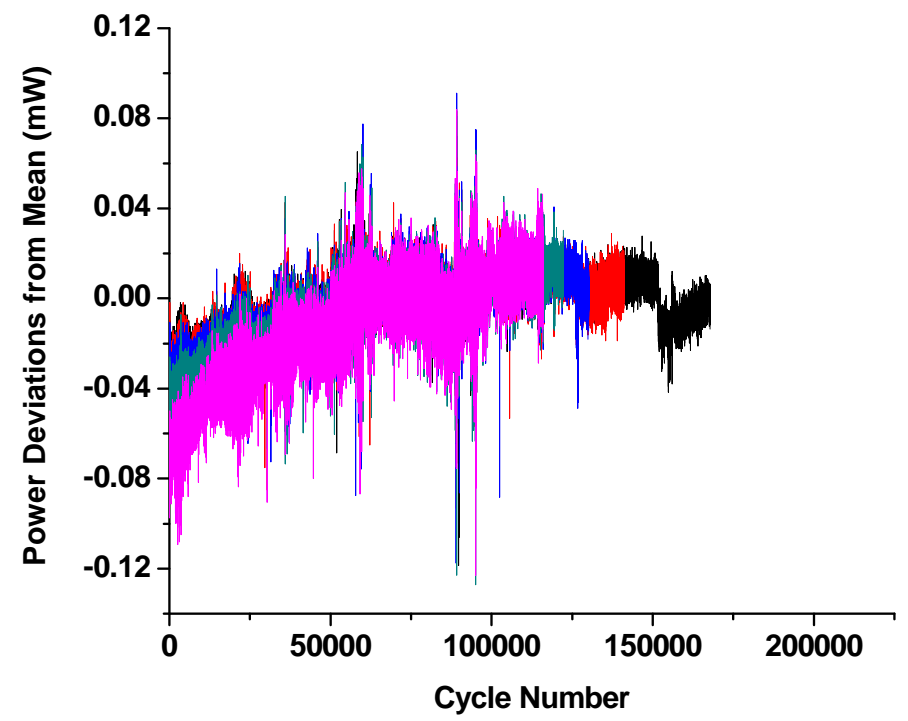

Figure 22. Power Deviations from the Mean Using Initial Zero Subtraction at Five Different Current Levels for a QC Laser Using a 100 kHz, 10\% Duty Cycle Current Modulation in Open Air Over Ten Months. The black, red, blue, dark cyan, and magenta traces represent the first through the fifth steps above threshold, respectively.

The data for all five levels show a rise in power over time similar to the Argos laser in Section 3.3.2. This rise in signal may be due to a burn-in effect, which causes an increase in power. Table 13 lists the mean power, standard deviations for the power readings, system and the laser, and twice the relative standard deviation for the laser for all five steps.

The data is fit with a first order exponential decay so that the time constant can be calculated. The individual time constants for each step are provided in Table 14 as a function of cycle number and operating time. The actual operating time of the laser is calculated by accounting for the $10 \%$ duty cycle as well as the 30 -second delay between cycles. Table 14 also lists the mean power calculated from the first 3000 cycles.

Table 13. Zero Subtracted Power Meter Readings and Standard Deviations for the Data Shown in Figure 22

\begin{tabular}{||l|c|c|c|c|c||}
\hline Average Power $(\mathrm{mW})$ & 0.563 & 1.161 & 1.750 & 2.989 & 5.564 \\
\hline Standard Deviation $(\mu \mathrm{W})$ & 12.2 & 13.4 & 15.4 & 18.4 & 24.4 \\
\hline Std. Dev. from Power Meter $(\mu \mathrm{W})$ & 3.0 & 3.0 & 3.6 & 4.9 & 6.2 \\
\hline Std. Dev. for Laser $(\mu \mathrm{W})$ & 11.8 & 13.0 & 14.9 & 17.7 & 23.5 \\
\hline $2 \times$ Rel. Std. Dev. of Laser & $4.2 \%$ & $2.2 \%$ & $1.7 \%$ & $1.1 \%$ & $0.8 \%$ \\
\hline
\end{tabular}


Table 14. Time Constants from the Exponential Fits to the Data Shown in Figure 22 as Well as the Average Power from the First 3000 Cycles

\begin{tabular}{||l|c|c|c|c|c||}
\hline Time Constant (cycle number) & 35644 & 36124 & 43186 & 51043 & 54592 \\
\hline Time Constant (hours operating) & 133 & 134 & 161 & 190 & 203 \\
\hline Average Power (mW) & 0.533 & 1.129 & 1.711 & 2.940 & 5.497 \\
\hline
\end{tabular}

Table 15. Zero Subtracted Power Meter Readings and Standard Deviations for the Data Shown in Figure 22 After Three Time Constants

\begin{tabular}{||l|c|c|c|c|c|}
\hline \hline Average Power $(\mathrm{mW})$ & 0.571 & 1.171 & 1.762 & 3.005 & 5.587 \\
\hline Change in Power $(\mathrm{mW})$ & .037 & .042 & .051 & .065 & .09 \\
\hline Standard Deviation $(\mu \mathrm{W})$ & 6.9 & 7.7 & 8.1 & 8.3 & 8.0 \\
\hline Std. Dev. from Power Meter $(\mu \mathrm{W})$ & 3.0 & 3.0 & 3.6 & 4.9 & 6.2 \\
\hline Std. Dev. for Laser $(\mu \mathrm{W})$ & 6.2 & 7.1 & 7.3 & 6.7 & 5.1 \\
\hline $2 \times$ Rel. Std. Dev. of Laser & $2.4 \%$ & $1.2 \%$ & $0.8 \%$ & $0.4 \%$ & $0.2 \%$ \\
\hline
\end{tabular}

The mean power is calculated for the data after three time constants using the time constant for the last step; thus, less than $5 \%$ of drift due to burn-in is present in these averages. Table 15 lists the mean power for all five levels after three time constants, the change in power from the first 3000 cycles, and the standard deviations for the mean, system, and laser.

Thus, very good results are obtained after the initial burn-in. For all of the steps, the fluctuations are on the order of $6-7 \mu \mathrm{W}$ resulting in stabilities that vary from $2 \%$ for the lowest level down to $0.2 \%$ for the highest level.

\subsection{Stability of Mid-Infrared Fiber Transmission}

Optical fibers have been used in the past for calibration systems (Anderson et al. 1992). However, optical fibers for the mid-infrared, and especially for the long-wave infrared ranging from 5 to $12 \mu \mathrm{m}$, are not as mature a technology as silica optical fiber, which cannot transmit light with wavelengths longer than $2 \mu \mathrm{m}$. Chalcogenide glass optical fibers are transparent from 2 to $9 \mu \mathrm{m}$ and are commercially available, making them an attractive solution. During FY06, multimode fibers were purchased and tested with a QCL in order to study the spatial uniformity of their output beam. It was shown that mechanically moving the fiber to scramble the propagating modes and averaging several frames with a camera lead to a significant increase in spatial uniformity.

However, it is also important to study the power stability over time of the laser when used with a fiber. The presence of the fiber in the system can add noise from different sources such as the accoustooptic effects in the fiber and optical feedback in the laser and could be detrimental to the calibration process. The stability of the laser by itself was characterized by using the conventional procedure typically used in the project; that is, repeatedly ramping the current in successive steps while recording 
the power. Three current steps are used: one below threshold that lasts 40 seconds and two above threshold that each last 20 seconds. The power during the last 13 seconds of a current step above threshold is averaged and logged, and the overall 80-second cycle is repeated, resulting in two power levels measured over time. A thermopile detector is used to measure the optical power and the detector and laser are shielded from air currents. In addition, the current source of the laser draws its power from an uninterruptable power supply, which minimizes the electrical power fluctuations that could lead to fluctuations in the current through the laser. The experiment was performed for three continuous days. The noise level is defined by the ratio of twice the standard deviation to the mean power at a given current step value. At low power, the noise level was $0.14 \%$ and at high power, $0.10 \%$. These values are comparable to long-term stability measurement results obtained previously in the project.

The laser output is coupled into the fiber by placing the fiber in close proximity to the laser facet. This simple technique has the advantage of not requiring any optical components and still provides a very high coupling coefficient close to $100 \%$ - without considering the Fresnel reflections of $21 \%$ at both fiber faces. The fiber absorption is negligible over the fiber length of $1 \mathrm{~m}$. The fiber core diameter is $100 \mu \mathrm{m}$ and its numerical aperture (NA) is 0.6. Both ends of the fiber are set in v-grooves to prevent their movement and a motor moves the fiber at the middle position of its length (Figure 23). The procedure is similar to the one presented earlier in the FY06 Final Report (Myers et al. 2006).

In a first series of experiments, the motor was not activated and the fiber was unperturbed. The power noise level was measured twice using the same protocol as before and yielded large noise values in the 3.40-4.53\% range, more than one order of magnitude larger than without the fiber. For a similar experiment where the fiber was moved, the noise level was reduced to $2.99 \%$ at low power and $2.47 \%$ at high power, but again more than an order of magnitude larger than without the fiber.
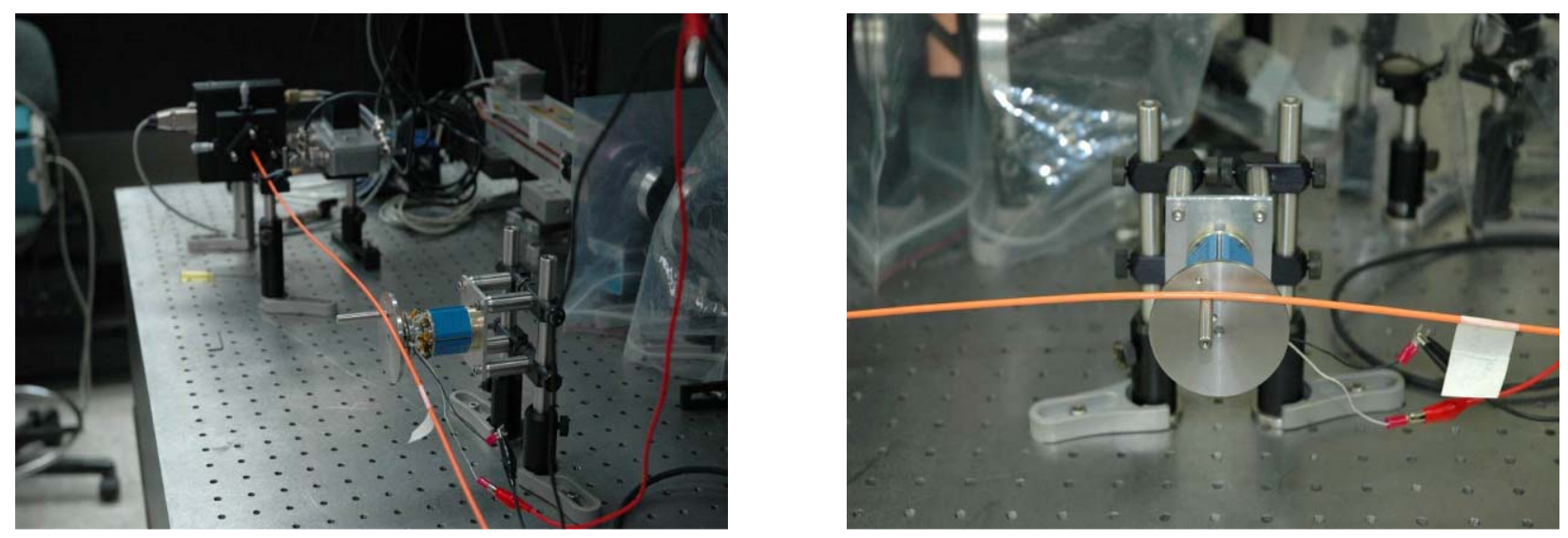

Figure 23. Photographs of the Multimode Chalcogenide Glass Optical Fiber Coupled to the QCL (left) and the Motor Used to Move the Middle of the Fiber Up and Down to Mix the Propagating Mode and Create a Uniform Output Beam (right). Figure reproduced from Myers et al. (2006). 
Table 16. Laser Amplitude Noise for Different Conditions

\begin{tabular}{||l|c|c||}
\hline & Noise at Low Power & Noise at High Power \\
\hline Laser & $0.14 \%$ & $0.10 \%$ \\
\hline Laser and Still Fiber 1 & $3.90 \%$ & $4.08 \%$ \\
\hline Laser and Still Fiber 2 & $4.53 \%$ & $3.40 \%$ \\
\hline Laser and Moving Fiber & $2.99 \%$ & $2.47 \%$ \\
\hline
\end{tabular}

Even though these results are fragmentary and more time needs to be devoted to the issue, they still reveal a large increase in noise when a fiber is used. QCL are known to be sensitive to optical feedback. When coupling the output of the QCL to the fiber, the fiber ends act as reflectors and send back a significant fraction of the power to the laser (21\% for each surface). Because the laser device is a FabryPerot cavity, several longitudinal modes can be excited and account for the overall power in different proportions. Optical feedback can disturb the relative proportion of power of the different modes, leading to amplitude noise. Moving the fiber can randomize the feedback from the end facet of the fiber and lower the noise value because light comes back through the fiber, but light reflected from the front facet is not randomized and still can cause significant feedback effects.

The precise mechanism accounting for the noise level when a fiber is added is still unconfirmed, but these simple experiments did show that using a fiber decreases the power output stability. The deposition of high-quality, anti-reflection coatings on the fiber facets could increase the stability, because the feedback would be significantly lower. Polishing the fiber ends at an angle would also reduce the feedback to the laser by reflecting the beam off-axis.

\subsection{Calibration System for a Microbolometer Array}

A bench-top system was built to demonstrate how a quantum cascade laser can be used to calibrate a microbolometer array. An Indigo infrared camera was used. Its array contains $320 \times 240$ pixels and it outputs 12-bit digital images. The camera is placed in front of a room-temperature QCL at a distance where the laser beam overfills the array of the camera, about $10 \mathrm{~cm}$ in this case. A personal computer running LabVIEW-based software is used to control the image acquisition and the current applied to the laser. Six different images are recorded for increasing incident laser power on the camera. So for every pixel, six data points (microbolometer response versus laser power) can be used to calibrate the pixel response by fitting a polynomial through the points. This way, all pixels are calibrated individually. The calibration is repeated several times after a suitable delay to highlight the response drift of the pixels over time. A key advantage of this calibration technique is that because several power levels are used, it is possible to correct for the potential nonlinearity of the pixel response.

A drawback of using a commercial camera is the fact that a protective window is placed in front of the microbolometer array. A highly coherent laser beam such as one produced by a QCL will create interference fringes in transmission through the window. To mitigate that problem, the camera is placed at a large angle $\left(\sim 40^{\circ}\right)$ with respect to the optical axis of the beam. Only a subset of 7170 pixels in a fringe-free region is calibrated. Figure 24 shows a typical image and the subset of pixels being calibrated. 


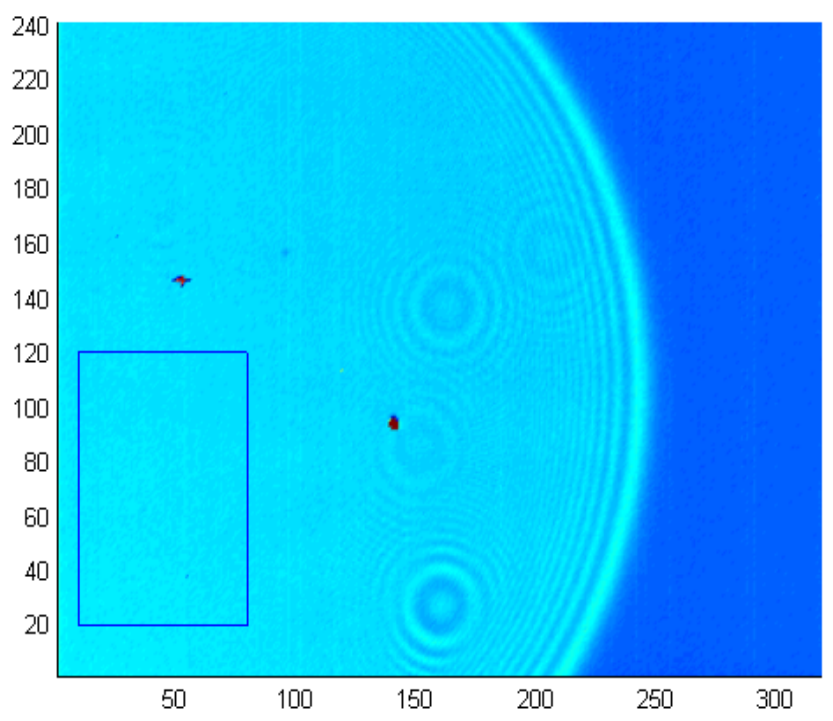

Figure 24. Typical Image from the Calibration System. The interference fringes are clearly seen on the right of the beam. The black dots are dead pixels and are irresponsive. The blue box shows the subset of pixels being calibrated.

The protocol used for the calibration is shown in Table 17. The laser current is kept to 0 when the system is not calibrating the array. Before the calibration starts, the laser current is raised to $1200 \mathrm{~mA}$, just below the lasing threshold, for 30 seconds in order to let the temperature of the laser settle to equilibrium at $15.0^{\circ} \mathrm{C}$. This step is necessary with the current laser used because of its very high threshold current ( 1225 mA). A better laser device having a low threshold would not need such a long thermalization time. After this step, the laser current is raised in 50-mA increments every 10 seconds. An image is recorded during the last $1 / 60^{\text {th }}$ of a second of the 10 -second delay, which is chosen to make sure the laser device has time to settle back to $15.0^{\circ} \mathrm{C}$. The temperature remains between $15.00 \pm 0.02^{\circ} \mathrm{C}$ when an image is being recorded. Six images are recorded for different current levels. For each of the 7170 pixels calibrated, a quadratic curve is fitted through the six pixel response versus laser current points.

The equation for fitting the pixel response is:

$$
y(x)=a_{0}+a_{1} x+a_{2} x^{2}
$$

where $y$ is the pixel response and $x$ is the current value, scaled from $-1<x<1$, in order to highlight the relative importance of the different terms with respect to each other. To be formally correct, the power values should be used instead of the current values, but because the current versus power relationship is close to linear over this range, the current values are used. 
Table 17. Calibration Scheme for the Array

\begin{tabular}{|l|l|}
\hline Laser Driving Current Levels (mA) & $\begin{array}{l}0-\text { between calibration steps } \\
1200-\text { Thermalization of QCL just below } \\
\text { threshold, no image recorded }\end{array}$ \\
& 1250 \\
& 1300 \\
& 1350 \\
& 1400 \\
& 1450 \\
& 1500 \\
\hline Thermalization Time (s) & 30.000 \\
\hline Time Between Calibration Current Levels (s) & 10.000 \\
\hline Time Between Calibrations (s) & 300.000 \\
\hline \hline
\end{tabular}

Table 18. Mean Response of the Calibrated FPA Pixels

\begin{tabular}{|l|c|c|c|}
\hline \multicolumn{2}{|l|}{} & Pixel-to-Pixel Mean & $\begin{array}{c}\text { Pixel-to-Pixel Standard } \\
\text { Deviation }\end{array}$ \\
\hline Offset & $a_{0}$ & 1010.3 & 28.6 \\
\hline Linear response & $a_{1}$ & 185.6 & 11.6 \\
\hline Quadratic response & $a_{2}$ & -4.8 & 16.2 \\
\hline
\end{tabular}

First, the results are presented for a single calibration, just after the camera does its internal calibration. Microbolometer arrays usually have an internal calibration mechanism that brings a shield in front of the array, blocking outside sources of radiation, and the signal from every pixel is reset to the same value, thus ensuring a uniform image. As time goes by, the signal from the pixels will drift, and eventually it will be necessary to perform the internal calibration again. Just after the internal calibration, the fit coefficients from Eq. (1), averaged over all the pixels, are as shown in Table 18.

Because $-1<x<1$, the amplitude of the coefficients can be compared, and it is clear that $a_{0}$, the offset term, is the largest, followed by $a_{1}$, the linear response of the pixels, and by $a_{2}$, the quadratic response of the pixels, which has a very large pixel-to-pixel standard deviation but is still not negligible. Pixels with the mean value of $a_{2}$ would have a maximum error of $1.3 \%$ of the range if only a linear calibration using only the endpoints were used. However, this maximum error increases to $3.1 \%$ and $5.7 \%$ for pixels where $a_{2}$ is respectively plus and minus one standard deviation from the mean. This highlights the need for a multi-point calibration: the nonlinearity of the detector could not be accurately calibrated using only a two-point calibration. Figure 25 shows the distribution of calibration coefficients for all selected pixels. The linear and quadratic response coefficients do not show a clear distribution of values, contrary to the offset coefficient, where the high and low values are grouped together in this case. 
(a)

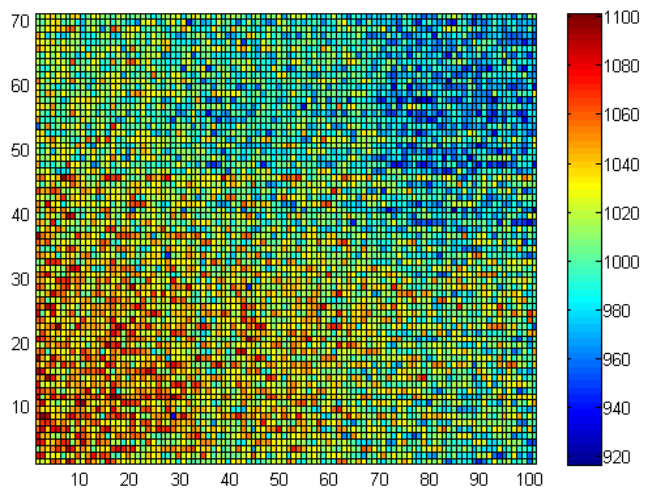

(c)

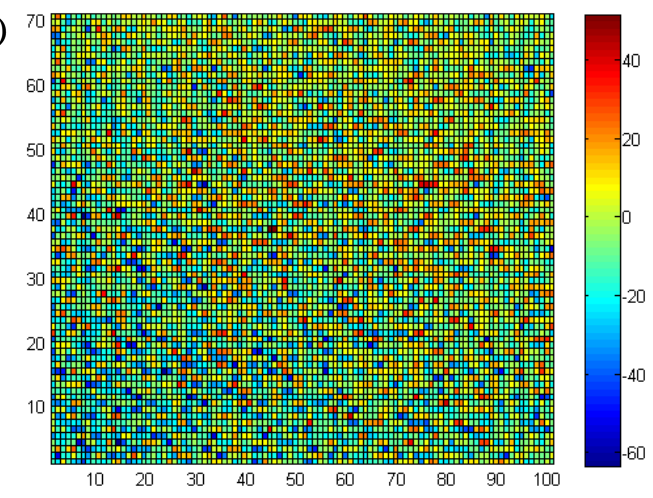

(b)

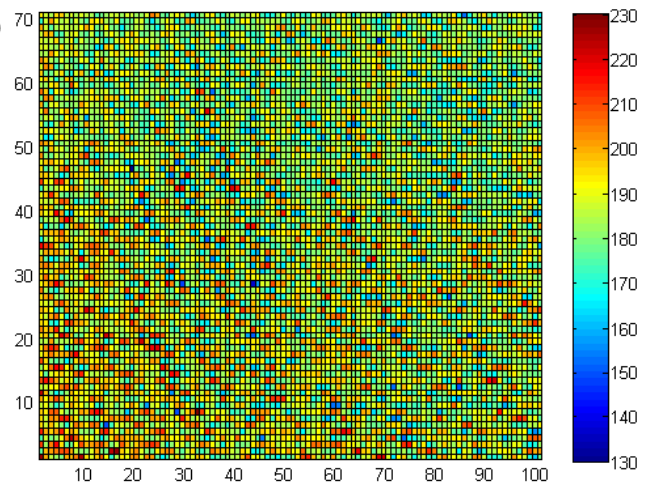

Figure 25. Distribution of Calibration Coefficients for Selected Pixels. (a) $a_{0}$ (b) $a_{1}$ (c) $a_{2}$.

The evolution of the calibration coefficients was also recorded as a function of time. In Figure 26, the time evolution of 10 pixels is shown. The offset coefficient goes up rapidly in the first hour, then stops drifting but still undergoes large fluctuations. The other coefficients do not have such a well-behaved trend, but do undergo large fluctuations as well.

To conclude, a calibration system using a QCL was developed and used to calibrate 7170 pixels of a microbolometer array. The six-point calibration is performed in under one minute and the points are fitted to a quadratic equation, allowing the characterization of the quadratic non-linearity of the detectors.

\subsection{Final Remarks}

We investigated the stability of the power meter used for the measurements presented in this report and constructed an inexpensive enclosure to provide a stable testing environment. Fluctuations on the order of $3 \mu \mathrm{W}$ are attributed to the power meter in this custom enclosure. A cw Fabry-Perot QCL in this set-up demonstrated mode-hop behavior with frequent power cycling so that better results were achieved with a modulated current waveform. With a modulated current, we demonstrated fluctuations less than $0.1 \%$ at various radiance levels. We plan to continue testing this laser and investigating and minimizing contributions from other noise sources such as the current supply in FY08. 

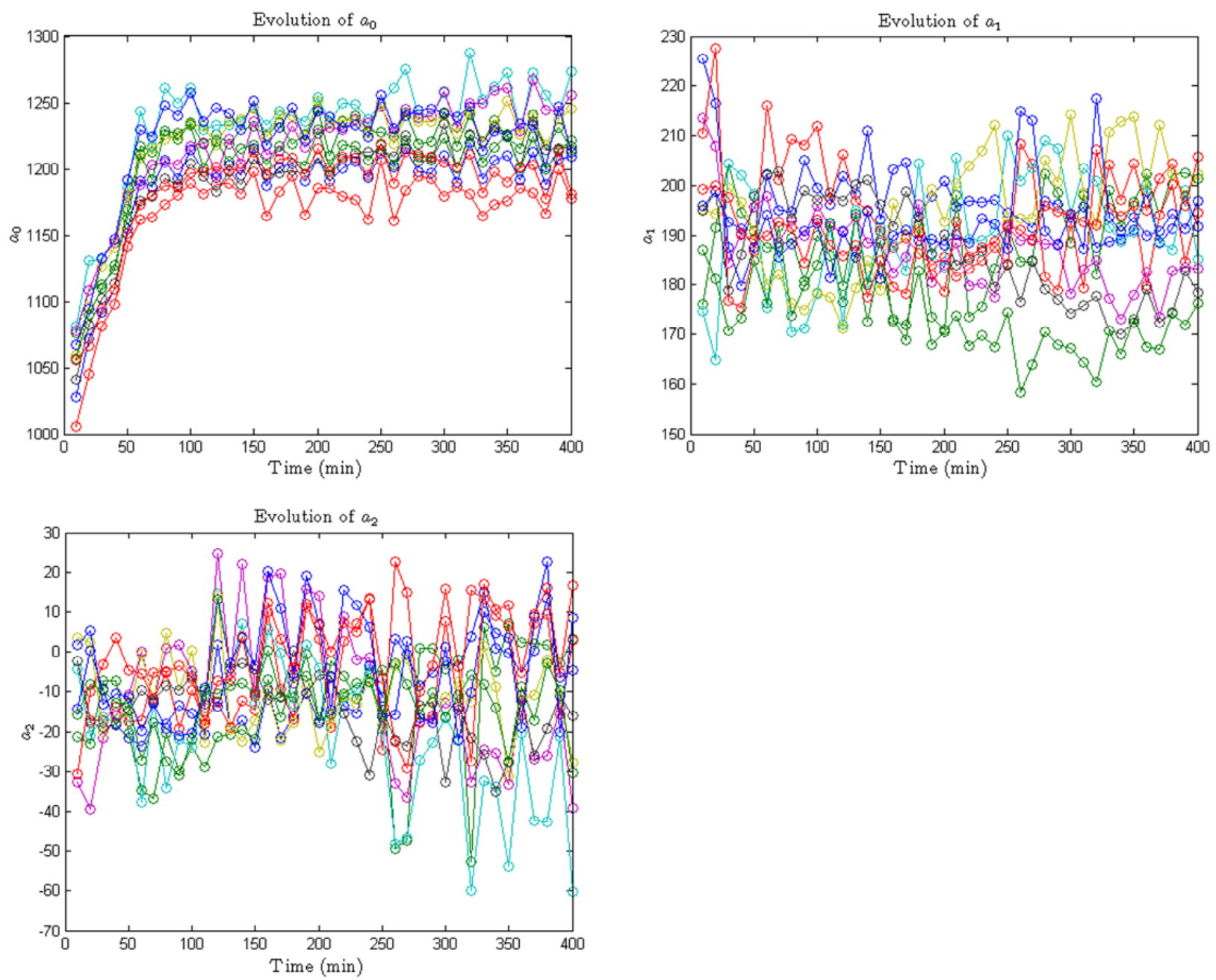

Figure 26. Time Evolution of the Calibration Coefficients

We also built a calibration system for a microbolometer array as a demonstration. We showed the multi-level radiance scheme could be used to calculate the calibration coefficients of the array. A quadratic fit was achieved on a fast timescale highlighting the merits of a multi-radiance calibration compared to a two-point check.

\subsection{References}

Anderson VE, NP Fox and DH Nettleton. 1992. "Highly stable, monochromatic and tunable optical radiation source and its application to high accuracy spectrophotometry,” Applied Optics 31, p.536-545.

Myers TL, BT Broocks, N Hô,and MC Phillips. 2006. Calibration Systems Development in FY06, PNNL-16296. Pacific Northwest National Laboratory, Richland, Washington. 


\section{Distribution}

No. of

Copies

OFFSITE

Dr. Victoria Franques

United States DOE

NNSA/NA-22

1000 Independence Ave. SW

Washington, DC 20585

Mr. Ralph Haller

15049 Conference Center Dr., Suite 600

Chantilly, VA 20151-3824
No. of

Copies

ONSITE

12 Pacific Northwest National Laboratory

Broocks, BT K5-25

Bruckner-Lea, C K5-25

Cannon, BD K5-25

Clemmer, RG K8-02

Myers, TL (5) K5-25

Sharpe, SW K8-88

Information Release Office (2) K1-06 


\section{Email notification only}

\section{OFFSITE}

Dr. Rhys M. Williams

United States DOE

NNSA/NA-22

1000 Independence Ave. SW

Washington, DC 20585

Dr. David Berry

United States DOE

NNSA/NA-22

1000 Independence Ave. SW

Washington, DC 20585

Mr. Ralph Hastings

United States DOE

NNSA/NA-22

1000 Independence Ave. SW

Washington, DC 20585

Mr. Eric Sander

United States DOE

NNSA/NA-22

1000 Independence Ave. SW

Washington, DC 20585

Mr. W. Randy Bell

United States DOE

NNSA/NA-22

1000 Independence Ave. SW

Washington, DC 20585 